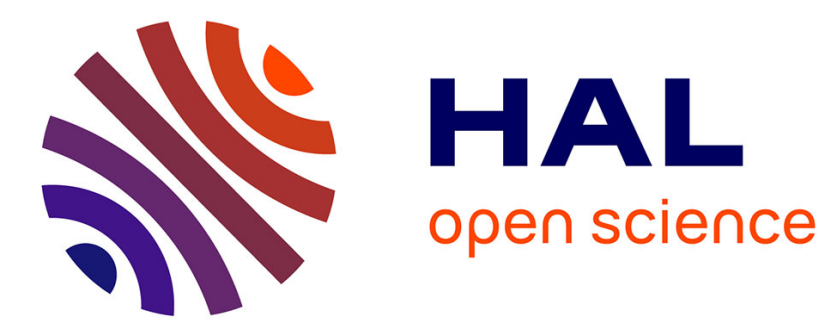

\title{
Système tolérant aux défauts: Synthèse d'une méthode de reconfiguration et/ou restructuration intégrant la fiabilité de certains composants
}

\author{
Fateh Guenab, Walter Schon, Jean-Louis Boulanger
}

\section{- To cite this version:}

Fateh Guenab, Walter Schon, Jean-Louis Boulanger. Système tolérant aux défauts: Synthèse d'une méthode de reconfiguration et/ou restructuration intégrant la fiabilité de certains composants. Journal Européen des Systèmes Automatisés (JESA), 2009, 43/10-2009, pp.1149-1178. hal-00447504

\author{
HAL Id: hal-00447504 \\ https://hal.science/hal-00447504
}

Submitted on 15 Jan 2010

HAL is a multi-disciplinary open access archive for the deposit and dissemination of scientific research documents, whether they are published or not. The documents may come from teaching and research institutions in France or abroad, or from public or private research centers.
L'archive ouverte pluridisciplinaire HAL, est destinée au dépôt et à la diffusion de documents scientifiques de niveau recherche, publiés ou non, émanant des établissements d'enseignement et de recherche français ou étrangers, des laboratoires publics ou privés. 


\title{
Système tolérant aux défauts : Synthèse d'une méthode de reconfiguration et/ou restructuration intégrant la fiabilité de certains composants
}

\author{
Fateh GUENAB, Walter SCHÖN et Jean-louis BOULANGER \\ Université de Technologie de Compiègne \\ Laboratoire Heudiasyc UMR CNRS 6599 \\ B.P. 20529, F-60205 COMPIEGNE \\ fateh.guenab@hds.utc.fr
}

\begin{abstract}
RÉSUMÉ. Le principe d'un système tolérant aux défauts (FTCS) est de préserver l'aptitude du procédé étudié à maintenir les performances les plus proches possibles de celles définies avant l'apparition de défauts. La contribution de notre travail réside dans le fait de proposer et de développer une stratégie de commande tolérante aux défauts (appelée communément reconfiguration ou restructuration), basée sur l'analyse de la fiabilité des composants et les performances du système. A l'issue de la détection et l'isolation d'un défaut dans le système, sous l'hypothèse qu'il existe plusieurs structures de fonctionnement résultant de la déconnexion des sous-systèmes défaillants ou leurs utilisations en mode dégradé, la tâche de reconfiguration consiste à déterminer parmi toutes les structures possibles celles qui assurent les performances initiales du système ou des performances dégradées. Dans un contexte multicritères, ce travail propose de déterminer la structure optimale en intégrant les performances du système et la fiabilité. L'approche proposée est illustrée sur un système hydraulique et thermique à 3 cuves.

ABSTRACT. The main goal of Fault Tolerant Control Systems (FTCS) is to preserve the ability of the system to achieve the objectives that has been assigned when faults or failures occurred. The main goal of this work is to develop a fault tolerant control strategy that incorporates both reliability and dynamic performance of the system for control reconfiguration. Once a fault has been detected and isolated, the reconfiguration strategy proposed in this paper tries to find possible structures of the faulty system that preserve prespecified performance, calculate the system reliability, compute new controller gains and finally search the optimal structure that has the "best" control performance with the highest reliability. The proposed approach is illustrated through a hydraulic and thermal system of three tanks.

MOTS-CLÉS: Systèmes Tolérants aux Défauts, Reconfiguration, Restructuration, Fiabilité des Systèmes, Méthode Pseudo-Inverse (PIM).

KEYWORDS: Fault Tolerant Control Systems, Control Reconfiguration, Restructuration, Systems Reliability, Pseudo-Inverse Method (PIM).
\end{abstract}

JESA. Volume $43-$ n$^{\circ} 10 / 2009$, pages 1149 à 1178 


\section{Introduction}

Depuis les trois dernières décennies, les systèmes industriels sont devenus de plus en plus complexes, utilisant des nouvelles technologies permettant d'accroître la qualité des produits et des services ainsi que la productivité des systèmes. En revanche, ces évolutions ont rendu les systèmes plus vulnérables aux défauts. Un défaut est un événement qui agit sur le système et qui peut changer les propriétés du système. Il peut modifier la structure du système et/ou ses paramètres, ou encore l'ensemble des lois de commande. Dans le but d'assurer la fiabilité des systèmes et la sécurité des humains, le problème de contrôle des systèmes en présence de défauts a été largement traité par plusieurs auteurs. La majeure partie des recherches a été consacrée au problème de la détection et la localisation de défauts de manière à déterminer l'état de fonctionnement du système (normal ou défaillant). Plusieurs approches sont utilisées pour résoudre ce problème, à titre d'exemple nous pouvons citer (Isermann, 1984), (Frank, 1990), (Patton et al., 1995), (Isermann, 1997) et (Chen et al., 1999).

Sous l'hypothèse d'un bloc de diagnostic fournissant les informations liées à la détection et à la localisation du défaut, il est possible soit de compenser l'effet du défaut (accommodation) soit de modifier les lois de commande de manière à amener le système dans un état le plus proche possible de celui dans lequel il se trouvait en fonctionnement normal (procédure appelée communément reconfiguration ou restructuration). Ces procédures à mettre en œuvre lors de l'occurrence du défaut ont été developpées sous forme de plusieurs stratégies regroupées sous l'expression «systèmes tolérants aux défauts» (en anglais, Fault Tolerant Control Systems FTCS). Un système de commande tolérant aux défauts est un système de commande qui permet de maintenir des performances proches de celles désirées tout en préservant la stabilité, non seulement en l'absence de défauts mais également en présence de composants défectueux. Si les performances initiales ne peuvent plus être garanties, un système FTCS doit assurer au moins des performances dégradées acceptables ou arrêter le système.

La commande tolérante aux défauts exploitant la fiabilité des systèmes est devenue un sujet très important. Plusieurs travaux ont été développés portant sur la fiabilité abordée sous plusieurs formes et avec différents objectifs. Certains chercheurs ont introduit la notion de la fiabilité du bloc diagnostic dans l'évaluation de la fiabilité du système complet sans établir un lien avec les lois de commande, voir à titre d'exemple (Bonivento et al., 2003), (Wu, 2001a) et récemment (Bonivento et al., 2006). Dans (Walker, 1997) les processus semi-markoviens ont été utilisés pour mesurer la fiabilité en définissant un modèle stochastique représentant l'occurrence des défauts, les décisions du bloc du diagnostic et les transitions entre ces états. Ce travail a donné lieu à un modèle complet de toutes les combinaisons entre les défauts possibles et de leurs détections sans tenir compte des relations dynamiques et des performances de la commande. Les auteurs de (Wu, 2001b) et (Wu, 2004) ont fait un lien entre la fiabilité du bloc diagnostic introduite dans (Wu, 2001a) et les lois de commande. Dans ces travaux, des modèles markoviens ont été utilisés pour évaluer la fiabilité des systèmes dont les sous-systèmes ont deux états de fonctionnement : normal ou en panne. Récemment (Li et al., 2006) ont proposé une méthode permettant d'évaluer la fiabilité 
des systèmes tolérants aux défauts en utilisant la modélisation par les chaînes de Markov. Une autre analyse de la fiabilité a été proposée par (Wu et al., 2002) où la fiabilité a été utilisée comme objectif à maximiser afin de sélectionner la structure optimale en termes de configuration. Le même esprit est présenté dans (Staroswiecki et al., 2004) où la fiabilité a été utilisée comme objectif à maximiser en assurant des objectifs en termes d'observabilité. En s'inspirant du travail de (Staroswiecki et al., 2004) et (Staroswiecki, 2000), les auteurs de (Figueras et al., 2006) ont proposé une méthode de synthèse d'un module diagnostic basée sur l'analyse de la fiabilité dans le but d'assurer des objectifs en termes de détectabilité des défauts. Récemment (Guenab et al., 2005) et (Guenab et al., 2006) ont proposé une méthode tolérante aux défauts intégrant la fiabilité et le coût financier. L'objectif est de déterminer une solution optimale pour la stratégie de reconfiguration, afin de maintenir des performances nominales ou au minimum dégradées. La solution se décline en termes de structure optimale garantissant des objectifs globaux exigés avec la fiabilité la plus élevée sous une contrainte de coût acceptable ou avec le moindre coût en respectant les limites de fiabilité nécessaires. Dans (Guenab et al., 2005) le choix de la structure dépend des fiabilités et des coûts financiers en prenant en compte uniquement les objectifs statiques, alors que dans (Guenab et al., 2006) les performances dynamiques ont été prises en compte.

Dans cet article, les performances statiques et dynamiques du système ainsi que les exigences de fiabilité sont prises en compte. Dans ce contexte, le système complexe est considéré comme un ensemble de sous-systèmes interconnectés, chacun peut assurer des objectifs locaux donnés en termes de fiabilité et de performances statiques et dynamiques. Chaque sous-système peut avoir plusieurs états de fonctionnement et plusieurs lois de commande spécifiques à son état. Dans le cas sans défaut, la structure du système, dite nominale, définit l'ensemble des sous-systèmes utilisés et des informations concernant leurs états et la manière dont ils sont interconnectés. Après l'occurrence du défaut, les sous-systèmes défaillants sont considérés comme capables de fonctionner dans des états dégradés permettant d'assurer des nouveaux objectifs locaux dégradés. De nouvelles structures du système peuvent être déterminées (en isolant, remplaçant les parties défaillantes ou les utilisant en mode dégradé). À chaque structure possible du système correspond une fiabilité et des performances globales calculées à partir des propriétés des sous-systèmes. Concernant la synthèse des lois de commande de chaque sous-système après l'occurrence du défaut, la méthode pseudoinverse (PIM), après modification et adaptation, a été utilisée pour illustrer le concept de la méthode de reconfiguration présentée dans cet article. La structure optimale correspond à la structure permettant d'assurer des objectifs globaux (statiques et dynamiques) avec une fiabilité maximale. Une fois que la solution optimale est fixée, une nouvelle structure et des nouvelles lois de commande pourraient être exploitées afin de maintenir les performances globales les plus proches possibles des nominales.

Cet article est organisé comme suit. Dans la section 2, une formulation générale du problème est présentée. La section 3 est consacrée à l'analyse de la fiabilité des systèmes. La méthode PIM est présentée dans la section 4. La section 5 est dédiée au développement de la stratégie de reconfiguration. Un exemple de simulation illustre la méthode proposée dans la section 6 . La dernière section est consacrée aux conclusions. 


\section{Contexte et problématique}

Une large classe de systèmes industriels peut être décrite par des structures hiérarchiques, appelées également systèmes multiniveaux. Les systèmes considérés dans notre travail sont des systèmes composés de deux niveaux : local et global (figure 1). Notons que l'extension à des systèmes multiniveaux ne représente pas de difficulté.

Au niveau local, plusieurs sous-systèmes sont généralement interconnectés, possédant chacun leur objectif local propre $\gamma_{l, i}$ avec $i=1 \ldots n$, ces objectifs locaux pouvant être regroupés pour assurer un objectif global $\gamma_{g}$. Le niveau supérieur, appelé coordinateur, prend en compte l'objectif global et les interactions entre les soussystèmes pour définir des objectifs locaux appropriés à chaque sous-système afin d'assurer l'objectif global.

Dans le présent travail, l'objectif local $\gamma_{l, i}$ est défini par l'objectif statique donné en termes de référence $r_{i}$ et les gains $K_{i}$ et $G_{i}$ des régulateurs locaux que nous aborderons dans la suite de l'article. L'objectif local est défini par $\gamma_{l, i} \equiv\left(r_{i}, K_{i}, G_{i}\right)$. L'objectif global $\gamma_{g}$ est défini en termes de référence globale $\gamma_{g, r e f}$. Notons que $\gamma_{g, r e f}$ représente une ou plusieurs références globales.

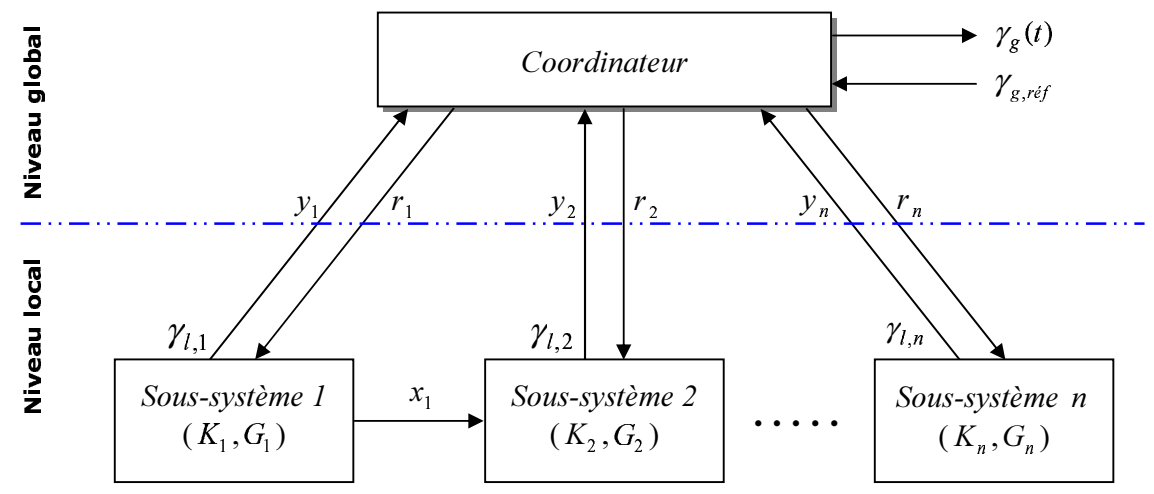

Figure 1. Architecture générale d'une structure hierarchique

Soit un système non linéaire représenté par le modèle suivant :

$$
\left\{\begin{aligned}
\dot{x}(t) & =f(x(t), u(t)) \\
y(t) & =g(x(t), u(t)) \\
\gamma_{g}(t) & =h(y(t))
\end{aligned}\right.
$$

où $f(x, u)$ et $g(x, u)$ sont les fonctions d'évolution d'état et de sortie. $h(y)$ représente l'évolution des objectifs globaux $\gamma_{g}$ en fonction des sorties $y$. Sachant que $y$ est l'ensemble des sorties $y_{i}$ des sous-systèmes et $u$ est l'ensemble des lois de commande.

Supposons que ce modèle non linéaire soit représenté par un système linéaire autour d'un point de fonctionnement donné. 
La représentation d'état du modèle linéaire est donnée par :

$$
\left\{\begin{aligned}
\dot{x}(t) & =A x(t)+B u(t) \\
y(t) & =C x(t)+D u(t) \\
\gamma_{g}(t) & =h(y(t))
\end{aligned}\right.
$$

La matrice $D$ est considérée nulle, car généralement la commande $u(t)$ n'a pas d'influence directe sur la sortie $y(t)$.

Le système décrit par l'équation [2] est composé de $n$ sous-systèmes $s_{i}, i=$ $1 \ldots n$, chacun est décrit par la représentation d'état suivante :

$$
\left\{\begin{aligned}
\dot{x}_{i}(t) & =A_{i} x_{i}(t)+B_{i} u_{i}(t)+\nu_{i}\left(t, x_{j}(t)\right) \\
y_{i}(t) & =C_{i} x_{i}(t)
\end{aligned}\right.
$$

$x_{i}$ est le vecteur d'état, $u_{i}$ est le vecteur de commandes, et $y_{i}$ est le vecteur de sorties du $i^{e}$ sous-système.

$\nu_{i}\left(t, x_{j}(t)\right)=\sum_{j=1, j \neq i}^{j=n} A_{i j} x_{j}(t)$ représente les interconnections entre le $i^{e}$ soussystème et les autres sous-systèmes " $j "$ avec $j=1 \ldots n$ et $j \neq i$.

$\nu_{i}\left(t, x_{j}(t)\right)$ est appelée la $i^{e}$ fonction d'interconnection.

Nous considérons que les sous-systèmes sont indépendants, au sens dynamique, dans ce cas la matrice $A$ est supposée diagonale et toutes les fonctions d'interconnections sont nulles. Dans le cas où $A$ est constituée de blocs diagonaux $A_{i}$, les soussystèmes sont alors décrits par :

$$
\left\{\begin{array}{l}
\dot{x}_{i}(t)=A_{i} x_{i}(t)+B_{i} u_{i}(t) \\
y_{i}(t)=C_{i} x_{i}(t)
\end{array}\right.
$$

Chaque sous-système $s_{i}$ possède son propre régulateur utilisant une loi de commande donnée sous la forme suivante :

$$
u_{i}(t)=-K_{i} x_{i}(t)+G_{i} r_{i}(t)
$$

Les gains $K_{i}$ et $G_{i}$ sont synthétisés de telle sorte que le sous-système en boucle fermée suit le modèle suivant :

$$
\begin{aligned}
& \dot{x}_{i}(t)=M_{i} x_{i}(t)+N_{i} r_{i}(t) \\
& \text { avec }\left\{\begin{array}{lll}
A_{i}-B_{i} K_{i}= & M_{i} \\
B_{i} G_{i} & = & N_{i}
\end{array}\right.
\end{aligned}
$$

\section{Problème de commande}

Le coordinateur se charge de résoudre le problème standard de commande (PSC) $<\gamma_{g, r e f}, \mathcal{S}, \theta, \mathcal{U}>$ qui consiste à déterminer : 
- l'ensemble des objectifs locaux en termes de références $r_{i}$ afin d'assurer les objectifs globaux statiques $\gamma_{g, r e f}$.

- l'ensemble des gains $K_{i}$ et $G_{i}$ afin d'assurer des performances dynamiques en termes de modèle (équations [6] et [7]).

La structure $\mathcal{S}$ définit l'ensemble des composants utilisés par le système et leur interconnexion. Elle regroupe les équations algébriques et différentielles représentant le comportement des composants du système.

Le PSC peut être réécrit sous la forme suivante :

- au niveau global, $<\gamma_{g, r e f}, \mathcal{S}, \theta, H\left(r_{i}\right)>$ consiste à déterminer les valeurs des références locales $r_{i}$ pour une fonction $h \in H$ dépendante de la structure $\mathcal{S}$ afin d'assurer les objectifs globaux $\gamma_{g \text {,ref }}$.

- au niveau local, le PSC regroupe l'ensemble des sous-problèmes locaux $<\mathcal{O}_{i}, \int_{i}, \theta_{i}, \mathcal{U}_{i}>$. où pour chaque sous-système $i,<\mathcal{O}_{i}, \int_{i}, \theta_{i}, \mathcal{U}_{i}>$ est définis par :

$$
\begin{aligned}
& \mathcal{O}_{i}: \quad \dot{x}_{i}(t)=M_{i} x_{i}(t)+N_{i} r_{i}(t) \quad \text { objectif local à assurer } \\
& \int_{i}: \dot{x}_{i}(t)=A_{i} x_{i}(t)+B_{i} u_{i}(t) \quad \text { contrainte en termes de structure } \\
& \mathcal{U}_{i}: u_{i}(t)=-K_{i} x_{i}(t)+G_{i} r_{i}(t) \quad \text { lois de commande admissibles }
\end{aligned}
$$

La solution de ces sous-problèmes consiste à trouver les gains $K_{i}$ et $G_{i}$.

Cas nominal : en l'absence de défauts, le système possède une structure nominale $\mathcal{S}^{\text {nom }}$, c'est le cas où le système utilise tous ses sous-systèmes et l'ensemble de leurs équations d'états décrites par la formule [4].

La solution nominale du problème $<\gamma_{g, r e f}^{\text {nom }}, \mathcal{S}^{\text {nom }}, \theta^{\text {nom }}, \mathcal{U}>$ est donnée par :

- la loi de commande nominale représentée par les lois de commande nominales locales définies par les gains nominaux $K_{i}^{\text {nom }}$ et $G_{i}^{\text {nom }}$ (équation [5]) calculés à partir des équations [4], [5] et [6] afin d'assurer les objectifs locaux (équation [6]).

- les objectifs locaux nominaux en termes de références $r_{i}^{\text {nom }}$ qui sont définis afin d'assurer les objectifs globaux nominaux statiques $\gamma_{g, r e f}^{\text {nom }}$.

Cas avec défaut : l'occurrence de défaut peut modifier la structure locale du soussystème (formule [4]) et par conséquent la structure du système complet (formule [2]). Le sous-système défaillant est alors décrit sous la forme suivante :

$$
\left\{\begin{array}{l}
\dot{x}_{i}(t)=A_{i}^{f} x_{i}(t)+B_{i}^{f} u_{i}(t) \\
y_{i}(t)=C_{i}^{f} x_{i}(t)
\end{array}\right.
$$

où $A_{i}^{f}, B_{i}^{f}$ et $C_{i}^{f}$ sont les matrices du $i^{e}$ sous-système en présence de défaut.

Notons que la stratégie présentée dans ce travail reste également valable pour les défauts système de commande et les défauts capteurs, mais seuls les défauts actionneurs sont considérés ici. Supposons que le défaut est détecté et estimé au niveau du $j^{e}$ actionneur. Ce défaut peut être modélisé de la manière suivante :

$$
u_{i j}^{f}=\left(1-\beta_{i j}^{f}\right) u_{i j}
$$


où $u_{i j}$ représente la $j^{e}$ composante du vecteur de commande du $i^{e}$ sous-système. Avec $\beta_{i j}^{f}=0$ dans le cas sans défaut, $\beta_{i j}^{f}=1$ représentant la perte totale d'actionneur et $\left.\beta_{i j}^{f} \in\right] 0,1\left[\right.$ une perte d'efficacité de $\beta_{i j}^{f} \%$.

La matrice $B_{i}^{f}$ est décrite comme suit $B_{i}^{f}=\operatorname{diag}\left(\left[1-\beta_{i j}^{f}\right]\right) B_{i}$.

Dans le cadre de notre travail, nous considérons que la reconfiguration du système peut être effectuée suivant plusieurs possibilités :

- remplacer le sous-système défaillant par un nouveau sous-système (redondance matérielle);

- utiliser le sous-système défaillant en mode dégradé et assurer le manque de sa fonction par d'autres sous-systèmes (redondance fonctionnelle);

- déconnecter le sous-système défaillant et continuer la mission avec les soussystèmes restants.

En conséquence, les objectifs locaux de chaque sous-système doivent être redéfinis afin d'assurer les objectifs globaux, dans le cas où ces derniers ne sont pas atteignables, des objectifs globaux dégradés peuvent être acceptés. Nous supposons que plusieurs structures peuvent exister, résultant de l'occurrence de défauts et des possiblités de reconfiguration mentionnées précédemment.

Sous l'hypothèse qu'il existe $M$ structures possibles $\mathcal{S}^{m}(m=1 \ldots M)$ et que chaque structure $\mathcal{S}^{m}$ contient $n_{m}$ sous-systèmes permettant d'assurer des objectifs globaux $\gamma_{g}^{m}$, le choix de la meilleure structure (optimale) $\mathcal{S}^{o p t}$ repose sur la définition des paramètres et informations à partir desquelles un critère doit être calculé.

Nous proposons d'effectuer le choix de la meilleure structure en nous basant sur l'analyse de la fiabilité des composants. Avant d'exposer une solution, nous présentons successivement le calcul de la fiabilité du système, la synthèse des lois de commande, le calcul des indices de performances statiques et de performance dynamiques (régulateurs) puis le calcul du critère du choix de la structure optimale.

\section{Analyse de la fiabilité}

\subsection{Estimation du taux de défaillance des composants}

D'après la norme AFNOR NF X 60-500, la fiabilité $R(t)$ est l'aptitude d'une entité à accomplir une fonction requise dans des conditions données pendant un intervalle de temps donné (AFNOR, 1988). L'analyse de la fiabilité des composants repose sur des données permettant de déduire le nombre de défaillances pendant une unité de temps (le taux de défaillance $\lambda$ ), quand le système dépend des conditions de fonctionnement normales. Pour les composants électroniques, dans la zone dite de vie utile, $\lambda$ est considéré constant. Généralement, lorsqu'un paramètre interne ou externe d'un composant varie, cela entraîne des modifications du taux de défaillance. Ces modifications sont dues à plusieurs causes : la technologie, les contraintes d'environnement et les conditions de fonctionnement. 
Dans plusieurs cas, le taux de défaillance est calculé pour différentes contraintes d'environnement, et pour différents niveaux de charges (puissance, tension,...). Dans le cadre de nos travaux, nous nous intéressons en particulier aux conditions de fonctionnement. Ces conditions représentent les modes de fonctionnement des composants, s'ils fonctionnent en continu ou non, et les niveaux des charges appliquées car elles peuvent changer d'une structure à l'autre.

L'objectif de cette sous-section est d'étudier l'impact des conditions de fonctionnement sur le taux de défaillance, nous cherchons à donner une relation analytique entre le mode de fonctionnement, en termes des charges appliquées, et le taux de défaillance.

Il existe plusieurs formules pour définir le taux de défaillance en fonction des conditions de fonctionnement (Martorell et al., 1999) et (Finkelstein, 1999). Nous utiliserons le modèle proportionnel de Cox, introduit par D.R. Cox. (PH : Proportional Hazards Model) (Cox, 1972) (Cox et al., 1984) et (Martorell et al., 1999).

Ce modèle suppose que le taux de défaillance est le produit d'un taux de défaillance de base (ou nominal) $\lambda_{0}(t)$ et une fonction $g(x)$ positive, qui interprète l'effet des paramètres de fonctionnement (température, puissance, tension ...)

$$
\lambda(t, x)=\lambda^{0}(t) \cdot g(x)
$$

$\lambda^{0}$ : taux de défaillance nominal (sous des conditions de fonctionnement nominales); $x$ : image de la charge directement dépendante de la commande $u$; $g(x)$ : fonction qui modélise l'effet des charges (stresses).

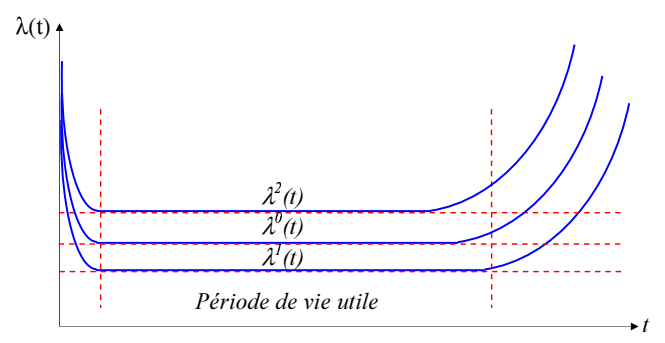

Figure 2. Taux de défaillance $\lambda^{0}(t), \lambda^{1}(t)$ et $\lambda^{2}(t)$

Pour illustration, la figure 2 représente le taux de défaillance nominal $\lambda^{0}(t)$ sous des conditions de fonctionnement nominales $\left(x_{0}\right)$ et les taux de défaillance $\lambda^{1}(t)$ et $\lambda^{2}(t)$ correspondants aux charges de valeurs différentes $x_{1}$ et $x_{2}$ avec $x_{1}<x_{0}<x_{2}$. Plusieurs définitions pour la fonction $g(x)$ peuvent être considérées, la forme exponentielle a été retenue pour cette étude du fait de sa simplicité : soit $g(x)=e^{x . \alpha}$, avec $\alpha$ constant. Notons que le taux de défaillance est considéré constant durant la période utile de la vie d'une structure $\mathcal{S}^{m}$, mais il dépend des conditions de fonctionnement de la structure. Le taux de défaillance du sous-système $i$ utilisé par la structure $\mathcal{S}^{m}$ est défini comme suit :

$$
\lambda_{i}^{m}\left(x_{i}^{m}\right)=\lambda_{i}^{0} \cdot e^{x_{i}^{m} \cdot \alpha_{i}}
$$


où $x_{i}^{m}$ est considérée constante durant la durée d'utilisation de la structure $\mathcal{S}^{m}$.

La charge $x_{i}^{m}$ appliquée au sous-système $i$, correspond à la commande $u_{i}^{m}$ ou une image de la commande au régime statique. Pour éclairer ce point, prenons l'exemple de la tension (en volts) appliquée sur une pompe pour délivrer un débit d'eau en $\mathrm{m}^{3} / \mathrm{s}$; la charge $x_{i}^{m}$ est le débit en $\mathrm{m}^{3} / \mathrm{s}$, en revanche dans le modèle analytique du système, la commande $u_{i}^{m}$ peut être le débit $\mathrm{m}^{3} / \mathrm{s}$ ou son équivalent en volts.

Dans le cas général, on considère la relation suivante :

$$
x_{i}^{m}=\eta_{i}^{m}\left(u_{i}^{m}(\infty)\right)
$$

Avec $\eta_{i}^{m}$ fonction permettant de définir la relation entre la charge et la commande en régime statique $u_{i}^{m}(\infty)$.

Le taux de défaillance donné par l'équation [11] est écrit comme suit :

$$
\lambda_{i}^{m}\left(u_{i}^{m}\right)=\lambda_{i}^{0} \cdot e^{\eta_{i}^{m}\left(u_{i}^{m}(\infty)\right) \cdot \alpha_{i}}
$$

\subsection{Calcul de la fiabilité}

Avant de présenter les formules de la fiabilité du système en fonction des fiabilités des sous-systèmes calculées à base des taux de défaillance suivant les conditions de fonctionnement de la structure $\mathcal{S}^{m}$, nous présentons le principe du calcul.

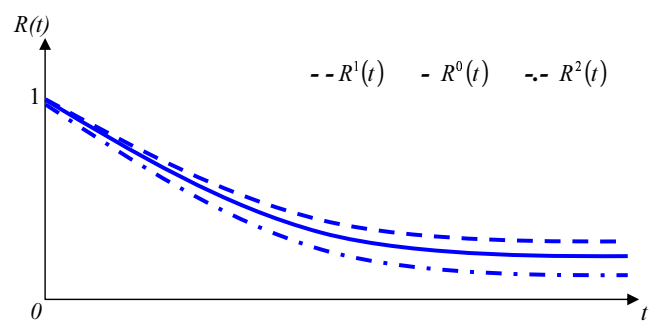

Figure 3. Fiabilités $R^{0}(t), R^{1}(t)$ et $R^{2}(t)$

La figure 3 illustre la fiabilité pour trois modes de fonctionnement : la fiabilité nominale $R^{0}(t)$ et les courbes de la fiabilité $R^{1}(t)$ et $R^{2}(t)$ calculées à partir des taux de défaillance pour les deux conditions de fonctionnement $\left(x_{1}\right.$ et $\left.x_{2}\right)$. Les fiabilités sont définies classiquement sous la forme exponentielle.

Supposons que le défaut apparaît à $t=t_{f}$, les conditions de fonctionnement étant nominales durant l'intervalle $\left[0, t_{f}\right]$ et que la nouvelle structure soit définie par le mode de fonctionnement qui correspond à la valeur de charge $x=x_{1}$ à partir de $t=t_{f}$ comme l'illustre la figure 4 .

La fiabilité entre $t=t_{f}$ et $\infty$ est donnée par la formule suivante :

$$
R(t)=e^{-\lambda^{0} \cdot t_{f}} \times e^{-\lambda^{1}\left(x_{1}\right) \cdot\left(t-t_{f}\right)}
$$




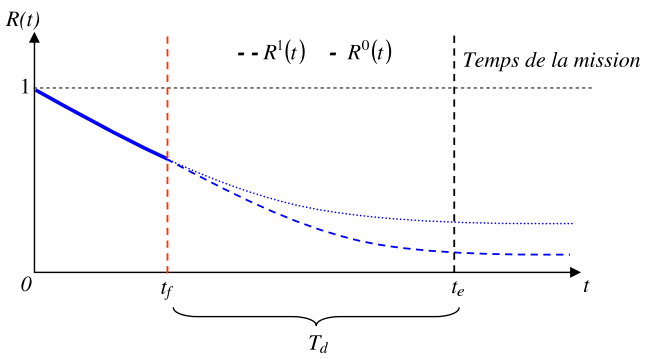

Figure 4. Fiabilité avant et après apparition du défaut

Et la fiabilité du système à la fin de sa mission (après une période de fonctionnement $T_{d}=t_{e}-t_{f}$ ) est définie comme suit :

$$
R\left(t_{e}\right)=R\left(t_{f}+T_{d}\right)=e^{-\lambda^{0} \cdot t_{f}} \times e^{-\lambda^{1}\left(x_{1}\right) \cdot\left(t_{e}-t_{f}\right)}
$$

Notons que l'instant d'occurrence de défaut $t_{f}$ est estimé en ligne et considéré constant durant toute la démarche d'optimisation.

À partir du taux de défaillance calculé dans l'équation [13], nous pouvons calculer la fiabilité $R_{i}^{m}\left(t_{e}, u_{i}^{m}\right)$ du sous-système $i$ utilisé par la structure $\mathcal{S}^{m}$ (conditions de fonctionnement définies par $u_{i}^{m}$ ) pour une période de temps donnée, notée $T_{d}$. La période $T_{d}$ se définit comme le temps de fonctionnement désiré avant la réparation des sous-systèmes défaillants (qui étaient la cause de la modification de la structure nominale) ou avant l'arrêt total du système. Dans la figure 4, c'est la période entre l'apparition du défaut $t_{f}$ et le temps de la mission du système $t_{e}$.

$R_{i}^{m}\left(t_{e}, u_{i}^{m}\right)$ est la fiabilité du sous-système $i$ utilisé par la structure $\mathcal{S}^{m}$ pour une période de temps donnée $T_{d}$ (entre $\left[t_{f}, t_{f}+T_{d}\right]$ ), donnée par :

$$
R_{i}^{m}\left(t_{e}, u_{i}^{m}\right)=e^{-\lambda_{i}^{0} \cdot t_{f}} \times e^{-\lambda_{i}^{m}\left(u_{i}^{m}\right) \cdot\left(t_{e}-t_{f}\right)}
$$

La fiabilité du système sous la structure $\mathcal{S}^{m}$ est calculée à partir de la fiabilité de ses $n_{m}$ sous-systèmes et dépend directement de leurs connexions (série, parallèle...). Sous l'hypothèse de l'indépendance causale des défaillances de chaque sous-système, si le système est composé de $n_{m}$ sous-systèmes en série; la fiabilité du système $R_{g}^{m}\left(t_{e}\right)$ est donnée par le produit des fiabilités de ses sous-systèmes.

$$
R_{g}^{m}\left(t_{e}, u_{i}^{m}\right)=\prod_{i=1}^{n_{m}} R_{i}^{m}\left(t_{e}, u_{i}^{m}\right)
$$

Pour des sous-systèmes en parallèle, la fiabilité $R_{g}^{m}\left(t_{e}\right)$ est donnée par :

$$
R_{g}^{m}\left(t_{e}, u_{i}^{m}\right)=1-\prod_{i=1}^{n_{m}}\left(1-R_{i}^{m}\left(t_{e}, u_{i}^{m}\right)\right)
$$

Et dans le cas mixte (série/parallèle), la fiabilité du système est calculée à partir des formules fondamentales [17] et [18]. 


\section{Méthode de la Pseudo-inverse (PIM)}

\subsection{Présentation de la méthode PIM}

La méthode de la pseudo-inverse, en anglais Pseudo-Inverse Method, a été traitée par plusieurs chercheurs (Ostroff, 1985), (Gao et al., 1990), (Gao et al., 1991). Elle a été présentée par (Huang et al., 1990) sous le nom approximate model matching.

La méthode PIM est utilisée dans le cas des systèmes linéaires. Elle consiste à déterminer un gain de retour d'état afin que la dynamique du système défaillant soit approximativement égale à celle du système nominal en minimisant un critère donné.

Soit le système linéaire nominal décrit par la représentation d'état suivante :

$$
\left\{\begin{array}{l}
\dot{x}(t)=A x(t)+B u(t) \\
y(t)=C x(t)
\end{array}\right.
$$

Le système est commandé par une loi de commande $u(t)=-K x(t)$ afin d'obtenir des performances désirées en boucle fermée. Le système bouclé est décrit par :

$$
\left\{\begin{array}{l}
\dot{x}(t)=(A-B K) x(t) \\
y(t)=C x(t)
\end{array}\right.
$$

Supposons que l'occurrence d'un défaut conduise à des changements du système décrit par la nouvelle forme d'état suivante :

$$
\left\{\begin{array}{l}
\dot{x}(t)=A^{f} x(t)+B^{f} u(t) \\
y(t)=C^{f} x(t)
\end{array}\right.
$$

Sous l'hypothèse que le système défaillant soit encore commandable, le problème consiste à trouver le gain $K^{f}$ permettant de maintenir les performances du système proches de celles désirées, conduisant à définir une nouvelle loi de commande :

$$
u^{f}(t)=-K^{f} x(t)
$$

afin que :

$$
A-B K=A^{f}-B^{f} K^{f}
$$

La solution est donnée par

$$
K^{f}=B^{f+}\left(A^{f}-(A-B K)\right)
$$

où $B^{f+}$ est la matrice pseudo-inverse de $B^{f}$, c'est pour cette raison que cette méthode est baptisée PIM.

Quand $B^{f}$ n'est pas de rank plein, les matrices $A^{f}-B^{f} K^{f}$ et $A-B K$ ne peuvent pas être égales, une solution approximative est alors calculée, en minimisant

$$
J=\left\|\left(A^{f}-B^{f} K^{f}\right)-(A-B K)\right\|_{F}^{2}
$$

où $\|\cdot\|_{F}$ est la norme Frobenius. 


\subsection{Limitations et extensions de la méthode PIM}

L'inconvénient majeur de la méthode classique (Ostroff, 1985), (Huang et al., 1990) réside dans le fait que trouver une solution $K^{f}$ qui minimise $J$ n'assure pas forcément la stabilité du système $A^{f}-B^{f} K^{f}$. La notion de proximité n'est pas précisément définie et le fait de trouver une solution minimisant le critère $J$, en d'autres termes, un système reconfiguré le plus proche possible du modèle nominal n'assure pas qu'il ait le même comportement dynamique.

La seconde limitation majeure est qu'il est nécessaire de connaître le modèle mathématique du système défaillant $A^{f}, B^{f}, C^{f}$ pour calculer le nouveau gain $K^{f}$.

Des extentions de la méthode ont été proposées par (Gao et al., 1991) et récemment par (Staroswiecki, 2005), ces extentions utilisent une optimisation sous contrainte pour garantir la stabilité de $A^{f}-B^{f} K^{f}$

Les auteurs de (Gao et al., 1991) ont proposé la méthode PIM modifiée MPIM. Elle est basée sur la méthode PIM classique en intégrant une méthode d'analyse de la stabilité du système avec des incertitudes dans ses paramètres. Nous avons constaté que cette solution permet d'assurer uniquement la stabilité du système après l'occurrence du défaut et sans maintenir les performances dynamiques du système nominal.

La solution proposée par (Staroswiecki, 2005) suppose que deux ensembles de matrices $\mathcal{M}$ et $\mathcal{N}$ peuvent être définies, tels que : $\forall(M, N) \in \mathcal{M} \times \mathcal{N}$, le modèle défini par $\dot{x}(t)=M x(t)+N e(t)$ possède un comportement dynamique acceptable.

La nouvelle formulation de la méthode PIM consiste à déterminer $\left(K^{f}, G^{f}\right)$, pour une certaine paire $(M, N) \in \mathcal{M} \times \mathcal{N}$, de façon que :

$$
A^{f}-B^{f} K^{f}=M \text { et } B^{f} G^{f}=N
$$

Les ensembles $\mathcal{M}$ et $\mathcal{N}$ sont définis hors ligne. Les calculs effectués en ligne consistent à déterminer $\left(K^{f}, G^{f}\right)$ et la paire $(M, N) \in \mathcal{M} \times \mathcal{N}$ pour laquelle l'équation [26] soit vérifiée. En revanche, l'auteur n'a pas proposé une méthode permettant de déterminer les ensembles $\mathcal{M}$ et $\mathcal{N}$, ni la paire $(M, N) \in \mathcal{M} \times \mathcal{N}$ pour laquelle l'équation [26] est vérifiée.

\subsection{Contribution à la synthèse de la méthode PIM modifiée}

Sur la base des travaux précédemment présentés (Gao et al., 1991) et (Staroswiecki, 2005), dans ce travail, nous proposons des modifications de la méthode PIM.

En mode nominal, le système bouclé (formule [27]) utilisant une loi de commande nominale $u(t)=-K^{\text {nom }} x(t)+G^{\text {nom }} r(t)$ suit correctement le modèle nominal décrit par la formule [28].

$$
\left\{\begin{array}{l}
\dot{x}(t)=\left(A-B K^{n o m}\right) x(t)+B G^{n o m} r(t) \\
y(t)=C x(t)
\end{array}\right.
$$




$$
\dot{x}(t)=M^{n o m} x(t)+N^{n o m} r(t)
$$

En d'autres termes, les équations suivantes sont vérifiées :

$$
\left\{\begin{aligned}
A-B K^{\text {nom }} & =M^{\text {nom }} \\
B G^{\text {nom }} & =N^{\text {nom }}
\end{aligned}\right.
$$

Nous supposons qu'il existe deux ensembles $\mathcal{M}$ et $\mathcal{N}$ permettant de définir un ensemble de performances acceptables dans lequel la stabilité du système est inclue.

Àprès l'apparition du défaut, le système défaillant devient :

$$
\left\{\begin{aligned}
\dot{x}(t) & =\left(A^{f}-B^{f} K^{n o m}\right) x(t)+B^{f} G^{n o m} r(t) \\
y(t) & =C^{f} x(t)
\end{aligned}\right.
$$

Nous considérons que les matrices $A^{f}$ et $B^{f}$ sont connues à partir des matrices $A$ et $B$ quand le sous-système défaillant est enlevé. Dans le cas où le sous-système défaillant n'est pas déconnecté, les matrices $A^{f}$ et $B^{f}$ doivent être estimées.

Le défaut apparu est moins sévère et ne nécessite pas une reconfiguration si la loi de commande nominale assure encore des performances acceptables définies par $\mathcal{M}$ et $\mathcal{N}$, c'est-à-dire que sous l'hypothèse de la synthèse de régulateur robuste, la reconfiguration n'est pas obligatoire si les équations suivantes restent vérifiées :

$$
\left\{\begin{aligned}
A^{f}-B^{f} K^{n o m} & =M \in \mathcal{M} \\
B^{f} G^{n o m} & =N \in \mathcal{N}
\end{aligned}\right.
$$

Dans le cas où les équations [31] ne sont pas vérifiées, une nouvelle loi de commande $u^{f}(t)=-K^{f} x(t)+G^{f} r(t)$ est obligatoire afin d'assurer les performances initialement définies par $\mathcal{M}$ ou au moins des performances dégradées. Donc nous cherchons $\left(K^{f}, G^{f}\right)$ en minimisant $J_{1}=\left\|A^{f}-B^{f} K^{f}-M^{n o m}\right\|_{F}^{2}$ et $J_{2}=\left\|B^{f} G^{f}-N^{n o m}\right\|_{F}^{2}$ avec les contraintes $A^{f}-B^{f} K^{f} \in \mathcal{M}$ et $B^{f} G^{f} \in \mathcal{N}$.

En premier lieu, nous écrivons les contraintes sous forme d'un ensemble de contraintes d'égalité et d'inégalité linéaires ou non linéaires, en d'autres termes, les deux ensembles $\mathcal{M}$ et $\mathcal{N}$ sous formes $\mathfrak{g}_{1}(M) \leq 0$ et $\mathfrak{g}_{2}(N) \leq 0$. Notons que $\mathfrak{g}_{1}(M)$ (respectivement $\mathfrak{g}_{2}(N)$ ) est une fonction ou un ensemble des fonctions linéaires ou non linéaires continues qui dépend des élements de la matrice $M$ (respectivement $N$ ).

Par conséquent, $A^{f}-B^{f} K^{f} \in \mathcal{M}$ signifie que $\mathfrak{g}_{1}\left(A^{f}-B^{f} K^{f}\right) \leq 0$ et $B^{f} G^{f} \in$ $\mathcal{N}$ signifie que $\mathfrak{g}_{2}\left(B^{f} G^{f}\right) \leq 0$.

Le problème classique devient un problème de minimisation sous contrainte avec des algorithmes et outils adaptés pour le résoudre. Soit à résoudre :

$$
\begin{aligned}
& \mid \begin{array}{c}
K_{o p t}^{f}=\underset{K^{f}}{\arg } \quad \min \left\|A^{f}-B^{f} K^{f}-M^{n o m}\right\|_{F}^{2} \\
\text { avec } \quad \mathfrak{g}_{1}\left(A^{f}-B^{f} K^{f}\right) \leq 0
\end{array} \\
& \mid \begin{array}{cc}
G_{o p t}^{f}= & \arg \quad \min \left\|B^{f} G^{f}-N^{n o m}\right\|_{F}^{2} \\
\text { avec } \quad \mathfrak{g}_{2}\left(B^{f} G^{f}\right) \leq 0
\end{array}
\end{aligned}
$$


Dans le cas où le problème [32] n'a pas de solution, nous considérons que des objectifs dégradés peuvent être acceptés. Ces performances dégradées sont représentées par les modèles dégradés $\mathcal{M}^{d}$ définis de la même manière que $\mathcal{M}$. Les fonctions des contraintes sont à remplacer par $\mathfrak{g}_{1}^{d}$ et $\mathfrak{g}_{2}^{d}$ associées à l'ensemble $\mathcal{M}^{d}$ et $\mathcal{N}^{d}$.

La figure 5 illustre $\mathcal{M}$ l'espace réalisable des performances nominales délimité par les contraintes $\mathfrak{g}_{1}$ et l'espace des performances dégradées $\mathcal{M}^{d}$ dans un cas simple à deux dimensions où les éléments des matrices varient dans $\mathbb{R}^{2}$. Pour simplifier l'explication, nous n'exposons que l'ensemble $\mathcal{M}, \mathcal{N}$ étant analysé la même manière.

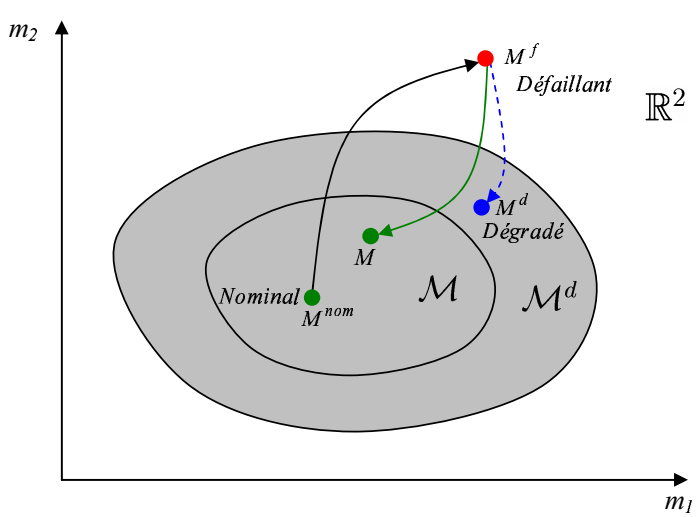

Figure 5. Espace réalisable des performances nominales $\mathcal{M}$ et des performances degradées $\mathcal{M}^{d}$

Le système est initialement en fonctionnement nominal $\left(M^{\text {nom }}\right)$ et suit correctement le modèle nominal décrit par $\dot{x}(t)=M^{n o m} x(t)$, le régulateur $K^{n o m}$ est synthétisé de façon que $A-B K^{\text {nom }}=M^{\text {nom }}$, à l'occurrence du défaut, le système passe en état défaillant décrit par la matrice $A^{f}-B^{f} K^{f}=M^{f}$, nous essayons de résoudre le problème [32], en trouvant $K_{o p t}^{f}$ qui minimise $J_{1}$ (solution très proche de $M^{\text {nom }}$ ) sous condition que $A^{f}-B^{f} K_{o p t}^{f}$ soit dans l'espace $\mathcal{M}$ ou au moins dans $\mathcal{M}^{d}$ dans le cas où il n'y a pas de solution dans $\mathcal{M}$.

La difficulté réside dans la définition des ensembles $\mathcal{M}$ et $\mathcal{N}$ pour représenter des performances bien définies. Dans le cadre de ce travail nous proposons une méthode pour déterminer l'ensemble $\mathcal{M}$ à partir du modèle nominal $M^{\text {nom }}$ par le biais des valeurs propres de chaque matrice $M \in \mathcal{M}$ dans un intervalle défini. Nous proposons également une méthode pour déterminer l'ensemble $\mathcal{N}$ à partir de la matrice $N^{n o m}$ permettant d'obtenir la sortie du système dans un intervalle autour de la référence. Nous renvoyons le lecteur à (Guenab, 2007) pour plus de détails.

\section{Exemple}

Soit un système décrit par un modèle de référence $\dot{x}(t)=M^{*} x(t)$ et $\tau_{1}^{*}=-1$, $\tau_{2}^{*}=-1,2$ et $\tau_{3}^{*}=-1,4$ les valeurs propres de $M^{*}$. Soit $\mathcal{M}$ l'ensemble des modèles admissibles ayant des valeurs propres $\tau_{1}^{*} \pm 10 \%, \tau_{2}^{*} \pm 10 \%$ et $\tau_{3}^{*} \pm 10 \%$. L'ensemble $\mathcal{M}$ a été définis en utilisant une méthode présentée dans (Guenab, 2007). 
$\mathcal{M}=\left\{M=\left[\begin{array}{lll}a & b & c \\ d & e & f \\ g & h & i\end{array}\right] \mid \begin{array}{r}-a-e-i-3.96 \leq 0 \\ a+e+i+3.24 \leq 0 \\ -b b f+a f h+g c e+d b i-a e i-d c h-2.2361 \leq 0 \\ b d-a i+g c-e i+e a-f h-5.1788 \leq 0 \\ g b f-a f h-g c e-d b i+a e i+d c h+1.2247 \leq 0\end{array}\right\}$

On peut vérifier que n'importe quelle matrice appartient à $\mathcal{M}$ possède des valeurs propres à $\pm 10 \%$ des valeurs propres nominales de $M^{*}$.

\section{Méthode proposée}

\subsection{Intégration de la méthode PIM modifiée dans la méthode de reconfiguration}

Pour rappel, soit l'architecture d'une structure $\mathcal{S}^{m}$ (figure 6), elle contient $n_{m}$ sous-systèmes, chacun étant modélisé par :

$$
\left\{\begin{array}{l}
\dot{x}_{i}^{m}(t)=A_{i}^{m} x_{i}^{m}(t)+B_{i}^{m} u_{i}^{m}(t) \\
y_{i}^{m}(t)=C_{i}^{m} x_{i}^{m}(t) \\
u_{i}^{m}(t)=-K_{i}^{m} x_{i}^{m}(t)+G_{i}^{m} r_{i}^{m}(t)
\end{array}\right.
$$

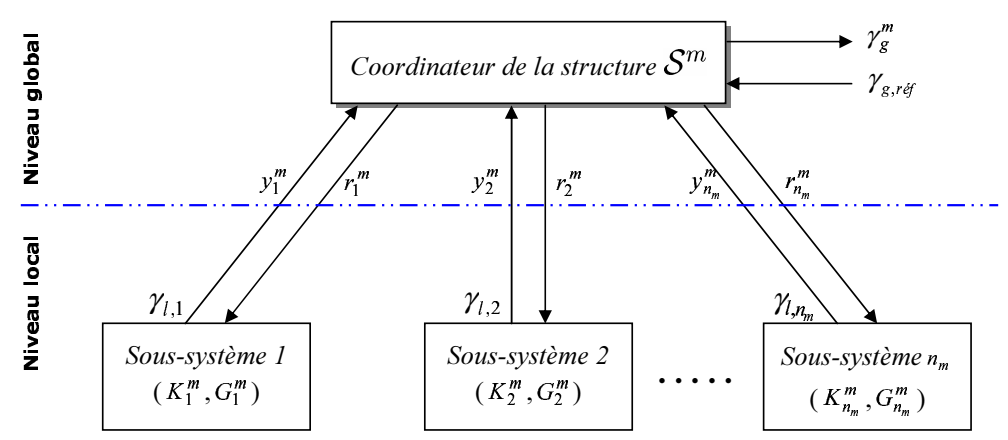

Figure 6. L'architecture du système sous une structure $\mathcal{S}^{m}$

Au niveau local d'une structure $\mathcal{S}^{m}$, considérons les sous-problèmes locaux donnés par $<\mathcal{O}_{i}^{m}, \int_{i}^{m}, \theta_{i}^{m}, \mathcal{U}_{i}^{m}>$, où pour chaque sous-système $i$ utilisé par la stucture $\mathcal{S}^{m}$, le problème donné par $<\mathcal{O}_{i}^{m}, \int_{i}^{m}, \theta_{i}^{m}, \mathcal{U}_{i}^{m}>$ est défini comme suit :

$$
\begin{array}{ll}
\mathcal{O}_{i}^{m}: \dot{x}_{i}^{m}(t)=M_{i}^{n o m} x_{i}^{m}(t)+N_{i}^{n o m} r_{i}^{m}(t) & \text { objectif local à assurer } \\
\int_{i}^{m}: \dot{x}_{i}^{m}(t)=A_{i}^{m} x_{i}^{m}(t)+B_{i}^{m} u_{i}^{m}(t) & \text { contrainte en termes de structure } \\
\mathcal{U}_{i}^{m}: u_{i}^{m}(t)=-K_{i}^{m} x_{i}^{m}(t)+G_{i}^{m} r_{i}^{m}(t) & \text { lois de commande admissibles }
\end{array}
$$

En intégrant les modifications de la méthode PIM de la sous-section 4.3 permettant d'assurer la stabilité du système, les problèmes $<\mathcal{O}_{i}^{m}, \int_{i}^{m}, \theta_{i}^{m}, \mathcal{U}_{i}^{m}>$ deviennent : 


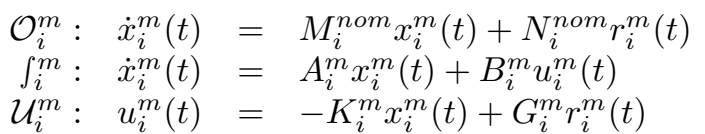

\begin{tabular}{l|rll} 
& $A_{i}^{m}-B_{i}^{m} K_{i}^{m}$ & $\in \mathcal{M}_{i}$ \\
$B_{i}^{m} G_{i}^{m}$ & $\in \mathcal{N}_{i}$
\end{tabular}

où les ensembles $\mathcal{M}_{i}$ et $\mathcal{N}_{i}$ représentent l'ensemble des modèles ayant des performances dynamiques acceptables pour chaque sous-système $i$. Ils sont donnés par :

$$
\begin{aligned}
\mathcal{M}_{i} & =\{M \quad|l| \\
\mathcal{N}_{i} & =\left\{N \mid \mathfrak{g}_{1, i}(M) \leq 0\right\} \\
& \left\{\begin{array}{l}
\left.\mathfrak{g}_{2, i}(N) \leq 0\right\}
\end{array}\right.
\end{aligned}
$$

Rappelons que $\mathfrak{g}_{1, i}(M)$ (respectivement $\mathfrak{g}_{2, i}(N)$ ) est une fonction ou un ensemble des fonctions linéaires ou non linéaires continues dépendant des élements de la matrice $M$ (respectivement $N$ ). Elles permettent d'écrire les ensembles $\mathcal{M}_{i}$ et $\mathcal{N}_{i}$ sous forme d'un ensemble de contraintes.

Après avoir défini les problèmes locaux, leurs solutions permettent de synthétiser les régulateurs $\left(K_{i}^{m}, G_{i}^{m}\right)$ de chaque sous-système. Les solutions sont données par :

$$
\begin{aligned}
& \mid \begin{array}{l}
K_{i, o p t}^{m}=\underset{i}{\arg } \quad \min \left\|A_{i}^{m}-B_{i}^{m} K_{i}^{m}-M_{i}^{\text {nom }}\right\|_{F}^{2} \\
\text { avec } \quad \mathfrak{g}_{1, i}\left(A_{i}^{m}-B_{i}^{m} K_{i}^{m}\right) \leq 0
\end{array} \\
& \mid \begin{array}{ll}
G_{i, \text { opt }}^{m}=\underset{G_{i}^{m}}{\arg } \min \left\|B_{i}^{m} G_{i}^{m}-N^{n o m}\right\|_{F}^{2} \\
\text { avec } & \mathfrak{g}_{2, i}\left(B_{i}^{m} G_{i}^{m}\right) \leq 0
\end{array}
\end{aligned}
$$

Les sous-systèmes bouclés utilisant les régulateurs $\left(K_{i, o p t}^{m}, G_{i, o p t}^{m}\right)$ sont donnés par :

$$
\left\{\begin{array}{l}
\dot{x}_{i}^{m}(t)=\left(A_{i}^{m}-B_{i}^{m} K_{i, o p t}^{m}\right) x_{i}^{m}(t)+B_{i}^{m} G_{i, o p t}^{m} r_{i}^{m}(t) \\
y_{i}^{m}(t)=C_{i}^{m} x_{i}^{m}(t)
\end{array}\right.
$$

Admettons que chaque sous-système $i$ possède $\tau_{j}^{m}$ valeurs propres avec $j=1 \ldots k_{i}$. Elles sont calculées à partir de la représentation donnée par l'équation [35].

\section{2. Évaluation des performances statiques}

Sachant que le rôle du système FTCS est de réduire ou limiter la différence entre la dynamique et la réponse statique du système nominal et le système reconfiguré, un indicateur de performances doit être défini pour choisir la meilleure structure du système reconfiguré. 
Comme nous l'avons indiqué à la section 2, chaque structure $m$ peut contenir $n_{m}$ sous-systèmes, chacun est modélisé par :

$$
\left\{\begin{array}{l}
\dot{x}_{i}^{m}(t)=A_{i}^{m} x_{i}^{m}(t)+B_{i}^{m} u_{i}^{m}(t) \\
y_{i}^{m}(t)=C_{i}^{m} x_{i}^{m}(t) \\
u_{i}^{m}(t)=-K_{i}^{m} x_{i}^{m}(t)+G_{i}^{m} r_{i}^{m}(t)
\end{array}\right.
$$

Et l'objectif global dépend des sorties des $n_{m}$ sous-systèmes comme suit :

$$
\gamma_{g}^{m}(t)=h^{m}\left(y_{i}^{m}(t)\right)
$$

avec $\left\{y_{i}^{m}(t) \quad \mid \quad i=1 \ldots n_{m}\right\}$ l'ensemble des sorties des sous-systèmes utilisés par le système sous la structure $\mathcal{S}^{m}$.

En régime statique, la valeur finale de l'objectif global, notée $\gamma_{g}^{m}$, est :

$$
\gamma_{g}^{m}(\infty)=\gamma_{g}^{m}=h^{m}\left(y_{i}^{m}(\infty)\right)
$$

où $y_{i}^{m}(\infty)$ sont les valeurs finales des sorties des sous-systèmes, elles sont calculées par le théorème des valeurs finales des sous-systèmes en boucle fermée :

$$
y_{i}^{m}(\infty)=\lim _{s \rightarrow 0} s y_{i}^{m}(s)=\lim _{s \rightarrow 0} T_{i}^{m}(s) \cdot r_{i}^{m}
$$

avec $T_{i}^{m}(s)$ la fonction de transfert du sous-système $i$ en boucle fermée :

$$
T_{i}^{m}(s)=C_{i}^{m}\left(I s-\left(A_{i}^{m}-B_{i}^{m} K_{i}^{m}\right)\right)^{-1} B_{i}^{m} G_{i}^{m}
$$

À partir des équations [38], [39] et [40], nous pouvons déduire une relation entre $\gamma_{g}^{m}$ et les références locales $r_{i}^{m}$ des sous-systèmes :

$$
\gamma_{g}^{m}=h^{m}\left(\left[C_{i}^{m}\left(-A_{i}^{m}+B_{i}^{m} K_{i}^{m}\right)^{-1} B_{i}^{m} G_{i}^{m}\right] \cdot r_{i}^{m}\right)
$$

Pour simplifier l'écriture, on note cette fonction dans la suite $\bar{h}^{m}$ telle que :

$$
\gamma_{g}^{m}=\bar{h}^{m}\left(r_{i}^{m}\right)
$$

L'indicateur normalisé est proposé pour évaluer les performances statiques de $\mathcal{S}^{m}$ :

$$
J_{\text {statique }}^{m}=\left|\frac{\gamma_{g}^{\text {nom }}-\gamma_{g}^{m}}{\gamma_{g}^{\text {nom }}}\right|
$$

où $\gamma_{g}^{\text {nom }}$ est l'objectif global du système nominal (sous sa structure nominale $\mathcal{S}^{\text {nom }}$ ) et $\gamma_{g}^{m}$ représente l'objectif global du système reconfiguré sous la structure $m$.

Dans cette sous-section, un indice de performances statiques a été proposé. Dans la sous-section suivante, nous allons proposer une évaluation des performances des régulateurs. 


\section{3. Évaluation des performances des régulateurs}

Pour des raisons de simplicité, nous supposons que l'image de la dynamique du système est comprise dans les valeurs propres. Nous cherchons à avoir des valeurs propres du système reconfiguré très proche de celles du système nominal. Soit l'écart normalisé entre les valeurs propres du $i^{\mathrm{e}}$ sous-système donné par :

$$
\varepsilon_{i}^{m}(j)=\left|\frac{\tau_{j}^{n o m}-\tau_{j}^{m}}{\tau_{j}^{n o m}}\right| \quad j=1 \ldots k_{i}
$$

où chaque sous-système $i$ possède $k_{i}$ valeurs propres $\tau_{j}^{\text {nom }}$ dans le cas nominal et $\tau_{j}^{m}$ dans le cas du système sous la structure $m$, avec $j=1 \ldots k_{i}$. Les valeurs propres $\tau_{j}^{m}$ sont calculées en ligne en utilisant l'équation [35].

L'écart maximal est donné par :

$$
\varepsilon_{i, \max }^{m}=\max _{j}\left(\varepsilon_{i}^{m}(j)\right) \quad j=1 \ldots k_{i}
$$

Sachant qu'une structure $\mathcal{S}^{m}$ est composée de $n_{m}$ sous-systèmes, nous calculons les écarts maximaux $\varepsilon_{i, \max }^{m}$ (équation [45]) pour les sous-systèmes $i$ avec $i=1 \ldots n_{m}$. Un indice permettant d'évaluer les performances dynamiques d'une structure $\mathcal{S}^{m}$ avec $m=1 \ldots M$ est défini par rapport au plus grand écart $\varepsilon_{i, \max }^{m}$, il est donné par :

$$
J_{d y n}^{m}=\max \left(\varepsilon_{i, \max }^{m}\right) \quad i=1 \ldots n_{m}
$$

L'indice $J_{d y n}^{m}$ peut être écrit sous la forme suivante :

$$
J_{d y n}^{m}=\max _{i}\left(\max _{j}\left(\left|\frac{\tau_{j}^{n o m}-\tau_{j}^{m}}{\tau_{j}^{n o m}}\right|\right)\right) \quad i=1 \ldots n_{m} \quad j=1 \ldots k_{i}
$$

\section{4. Étapes de la méthode proposée}

Sous la base des éléments précédemment définis, la solution optimale au problème considéré est obtenue en suivant les deux étapes suivantes :

Étape A) La première étape consiste à :

A.1) Déterminer les références locales optimales $r_{i, o p t}^{m}$ des sous-systèmes de chaque structure $\mathcal{S}^{m}$ permettant d'assurer des meilleurs objectifs statiques globaux, en minimisant l'indice de performance $J_{\text {statique }}^{m}$ sous contraintes d'une fiabilité minimale, soit $R_{g}^{m}\left(t_{e}\right) \geq R_{g}^{*}$ avec $R_{g}^{*}$ correspond à un seuil à ne pas dépasser.

A.2) Calculer l'indice des performances statiques globales $J_{\text {statique (équation }}^{m}$ [43]) permettant d'évaluer les performances globales $\gamma^{m}$ de chaque structure $\mathcal{S}^{m}$.

A.3) Calculer les gains des régulateurs optimaux $\left(K_{o p t, i}^{m}, G_{o p t, i}^{m}\right)$ (équation [34]).

A.4) Calculer les indices des performances des régulateurs $J_{d y n}^{m}$ (équation [47]).

Le problème (A.1) consiste à résoudre un problème d'optimisation sous contrainte d'inégalité. Notons que dans la plupart des cas la solution optimale trouvée n'est pas unique. Généralement, le problème peut être écrit sous cette forme : 


\begin{tabular}{|lll} 
trouver & $x$ & variables \\
minimisant & $f(x)$ & fonction à optimiser \\
avec & $g(x) \leq 0$ & $q$ contraintes \\
et & $x \in \mathbb{R}^{l}, \quad g(x) \in \mathbb{R}^{q}$ &
\end{tabular}

où $\mathbb{R}^{l}$ est l'espace de recherche représentant l'ensemble des valeurs pouvant être prises par les variables $x=\left(x_{1}, x_{2} \ldots x_{l}\right)$.

L'ensemble des $q$ contraintes $\left(g=\left(g_{1}, g_{2} \ldots g_{q}\right)^{T}\right)$ permet de définir l'espace réalisable $\mathcal{X}$. Le problème consiste à trouver $x_{\text {opt }} \in \mathcal{X}$ permettant de minimiser $f$.

Le problème (A.1) s'écrit comme suit :

$$
\mid \begin{array}{ll}
\operatorname{minimiser} & \mathfrak{h}^{m}\left(r_{i}^{m}\right)=J_{\text {statique }}^{m}\left(r_{i}^{m}\right) \\
\text { avec } & \mathfrak{g}^{m}\left(r_{i}^{m}\right)=R_{g}^{*}-R_{g}^{m}\left(t_{e}\right) \leq 0
\end{array}
$$

$-\mathfrak{h}^{m}\left(r_{i}^{m}\right)$ est la fonction à minimiser;

- $\mathfrak{g}^{m}\left(r_{i}^{m}\right)$ une fonction représentant la contrainte de la fiabilité.

L'espace de recherche est défini par les bornes inférieure et supérieure des références notées $r_{i, \min }^{m}$ et $r_{i, \max }^{m}$ respectivement.

\section{La fonction à minimiser :}

En remplaçant l'équation [42] dans l'équation [43], on peut écrire la relation entre l'indicateur de performances statiques et les références locales $r_{i}^{m}$ :

$$
J_{\text {statique }}^{m}=\left|\frac{\gamma_{g}^{\text {nom }}-\bar{h}^{m}\left(r_{i}^{m}\right)}{\gamma_{g}^{\text {nom }}}\right|=\left|1-\frac{\bar{h}^{m}\left(r_{i}^{m}\right)}{\gamma_{g}^{\text {nom }}}\right|=\mathfrak{h}^{m}\left(\gamma_{g}^{\text {nom }}, r_{i}^{m}\right)
$$

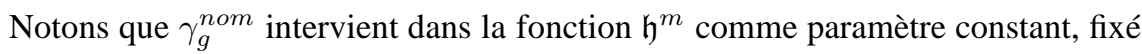
par l'opérateur. Puisqu'il n'est pas une variable à optimiser et pour des raisons de simplification d'écriture, on peut l'enlever de l'écriture mais en réalité il intervient implicitement dans les calculs. La formule précédente [49] devient :

$$
J_{\text {statique }}^{m}=\mathfrak{h}^{m}\left(r_{i}^{m}\right)
$$

\section{La contrainte de la fiabilité :}

Pour trouver une relation entre la fiabilité du système $R_{g}^{m}\left(t_{e}, u_{i}^{m}\right)$ sous la structure $\mathcal{S}^{m}$ et les références $r_{i}^{m}$ on utilise les équations précédentes de la manière suivante :

- à partir des formules [17] et [18] on écrit la relation :

$$
R_{g}^{m}\left(t_{e}, u_{i}^{m}\right)=\nabla^{m}\left(R_{i}^{m}\left(t_{e}, u_{i}^{m}\right)\right)
$$

La fonction $\nabla^{m}$ est utilisée pour exprimer la fiabilité du système en fonction des fiabilités de ses sous-systèmes, elle est donnée par l'équation [17] ou par l'équation [18] ou par une combinaison de ces équations suivant l'interconnexion des sous-systèmes (série, parallèle, mixte...).

- à partir des équations [36] en appliquant le théorème des valeurs finales, on peut définir la fonction $F^{m}\left(r_{i}^{m}\right)$ comme suit :

$$
u_{i}^{m}(\infty)=F^{m}\left(r_{i}^{m}\right)=\left(-A_{i}^{m}+B_{i}^{m} K_{i}^{m}\right)^{-1} B_{i}^{m} G_{i}^{m} r_{i}^{m}+B_{i}^{m} G_{i}^{m} r_{i}^{m}
$$


- remplaçant l'équation [52] dans l'équation [51], on peut déduire une relation entre la fiabilité du système sous la structure $\mathcal{S}^{m}$ et les références $r_{i}^{m}$ de l'ensemble de ses $n_{m}$ sous-systèmes :

$$
R_{g}^{m}\left(t_{e}, u_{i}^{m}\right)=\nabla^{m}\left(R_{i}^{m}\left(t_{e}, F^{m}\left(r_{i}^{m}\right)\right)\right)
$$

Finalement la contrainte de fiabilité est donnée par :

$$
\mathfrak{g}^{m}\left(r_{i}^{m}\right)=R_{g}^{*}-\nabla^{m}\left(R_{i}^{m}\left(t_{e}, F^{m}\left(r_{i}^{m}\right)\right)\right)
$$

Possédant la fonction à minimiser et les différentes contraintes, il suffit de résoudre le problème en utilisant l'un des algorithmes classiques de l'optimisation. Dans ce papier, nous avons utilisé la fonction fmincon de la boîte à outils Optimization Toolbox du logiciel MATLAB de mathworks.

\section{Étape B)}

La deuxième étape permet la sélection de la structure optimale $\mathcal{S}^{\text {opt }}$ parmi toutes les structures. Nous définissons un indice de performances regroupant les deux indices de performances statiques globales $J_{\text {statique }}^{m}$ et des régulateurs locaux $J_{d y n}^{m}$ comme suit :

$$
\mathcal{J}^{m}=\rho J_{\text {statique }}^{m}+(1-\rho) J_{\text {dyn }}^{m}
$$

Les indices $J_{\text {statique }}^{m}$ et $J_{d y n}^{m}$ sont pondérés par les poids $\rho$ et $(1-\rho)$ respectivement, ceci dans le but de favoriser l'une à l'autre des performances.

Finalement, la meilleure structure $\mathcal{S}^{\text {opt }}$ est déterminée de la manière suivante :

$$
\mid \begin{array}{ll}
\mathcal{S}^{\text {opt }}= & \arg \min \left(\mathcal{J}^{m}\right) \\
\text { avec } & m=1 \ldots M
\end{array}
$$

Après avoir présenté les étapes de la méthode proposée, nous allons illustrer et valider les résultats trouvés sur le processus hydraulique et thermique suivant.

\section{Application}

La méthode que nous avons présentée est appliquée sur un processus hydraulique et thermique. Le système est représenté par la figure 7. L'objectif global est de conserver un volume de fluide constant à une température désirée au sein d'une cuve. Le système est composé de trois cuves cylindriques de section identique $S$. Deux cuves sont utilisées pour chauffer les liquides issus de deux pompes. Les températures des liquides sont ajustées par des résistances électriques. Les sections des tuyaux sont identiques $S_{1}=S_{2}$ et les cœefficients de viscosité du liquide sont $\mu_{1}=\mu_{2}$. La troisième cuve sert à mélanger les liquides. Le système est composé de quatre actionneurs et six capteurs. Les signaux de commande sont $p_{1}, p_{2}$ les puissances délivrées par les deux résistances et $q_{1}, q_{2}$ les débits fournis par les deux pompes. Les mesures sont les températures $\left(t_{1}, t_{2}, t_{3}\right)$ et les niveaux de liquide $\left(h_{1}, h_{2}, h_{3}\right)$ dans chaque cuve. 


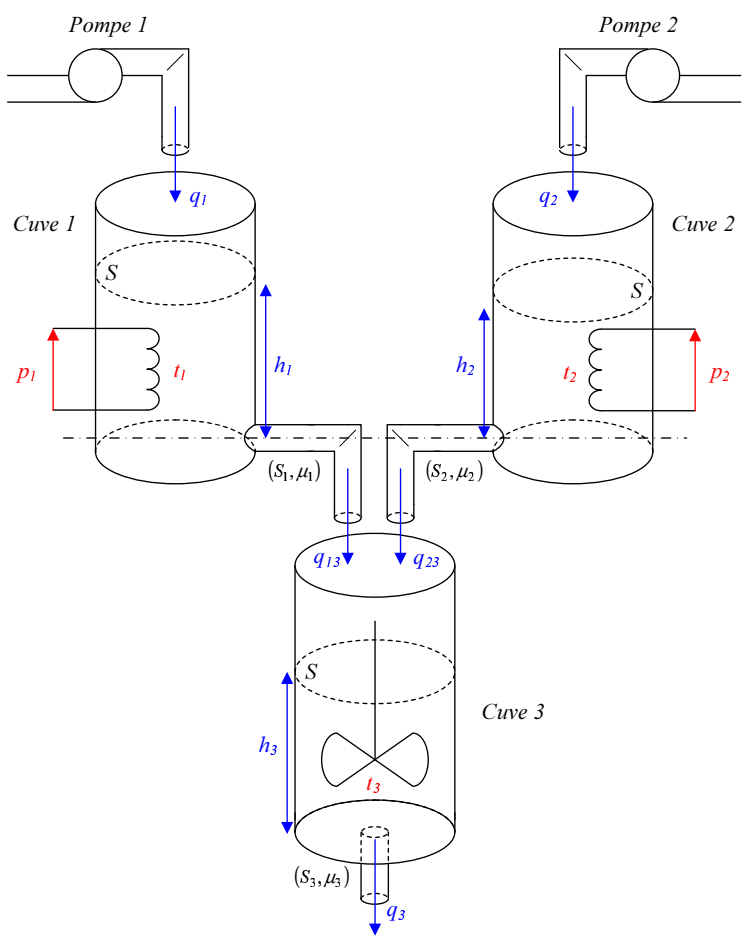

Figure 7. Schématisation du système

En effectuant le bilan volumique et le bilan calorimétique, nous obtenons la représentation non linéaire du système :

$$
\begin{aligned}
& \left\{\begin{array}{l}
\dot{h_{1}}(t)=\frac{1}{S}\left(q_{1}(t)-\alpha_{1} \sqrt{h_{1}(t)}\right) \\
\dot{h_{2}}(t)=\frac{1}{S}\left(q_{2}(t)-\alpha_{2} \sqrt{h_{2}(t)}\right) \\
\dot{h_{3}}(t)=\frac{1}{S}\left(\alpha_{1} \sqrt{h_{1}(t)}+\alpha_{2} \sqrt{h_{2}(t)}-\alpha_{3} \sqrt{h_{3}(t)}\right)
\end{array}\right. \\
& \left\{\begin{array}{l}
\dot{t_{1}}(t)=\frac{1}{S h_{1}(t)}\left(\frac{p_{1}(t)}{\mu c}-\left(t_{1}-t_{1_{i}}\right) q_{1}(t)\right) \\
\dot{t_{2}}(t)=\frac{1}{S h_{2}(t)}\left(\frac{p_{2}(t)}{\mu c}-\left(t_{2}-t_{2_{i}}\right) q_{2}(t)\right) \\
\dot{t_{3}}(t)=\frac{-1}{S h_{3}(t)}\left(q_{13}(t)\left[t_{3}(t)-t_{1}(t)\right]+q_{23}(t)\left[t_{3}(t)-t_{2}(t)\right]\right)
\end{array}\right.
\end{aligned}
$$

Sachant que le système réel est non linéaire, sa représentation par un modèle linéaire simple n'est valable que dans un domaine limité. Afin d'obtenir une représentation du comportement du système dans un large domaine, nous le représentons par la combinaison d'un nombre fini de modèles simples linéaires, appellés modèles locaux. Chaque modèle est valable au voisinage d'un point de fonctionnement donné. Une 
fonction d'activation permet d'indiquer la validité du modèle local sur un domaine donné. Sa valeur dépend du vecteur d'états et de commande du système, elle vaut 1 (ou voisine de 1) sur le domaine de validité du point de fonctionnement et tend rapidement vers zéro en dehors de ce domaine. Une fonction gaussienne peut être choisie à cet effet. Dans le cas le plus simple, la représentation globale du système est donnée par la somme des modèles locaux pondérés par leurs fonctions d'activations. Pour plus de détails, nous renvoyons le lecteur à (Murray-Smith et al., 1997).

Pour obtenir le multimodèle, il faut d'abord linéariser le système autour d'un point de fonctionnement $\left(h_{1_{0}}, h_{2_{0}}, h_{3_{0}}, t_{1_{0}}, t_{2_{0}}, t_{3_{0}}, q_{1_{0}}, q_{2_{0}}, p_{1_{0}}, p_{2_{0}}\right)$ ensuite nous allons choisir les valeurs de points de fonctionnement et définir les fonctions d'activation.

En linéarisant les équations [57] et [58], le système peut être écrit sous la forme :

$$
\left\{\begin{array}{l}
\dot{X}(t)=A X(t)+B U(t) \\
Y(t)=C X(t)+D U(t)
\end{array}\right.
$$

avec $X(t)=\left[h_{1} t_{1} h_{2} t_{2} h_{3} t_{3}\right]^{T}, U(t)=\left[q_{1} p_{1} q_{2} p_{2}\right]^{T}$ et $Y(t)=X(t)$.

La matrice $C$ est la matrice d'identité $[6 \times 6]$ et $D$ est la matrice nulle $[6 \times 4]$.

Les matrices $A$ et $B$ sont successivement données par :

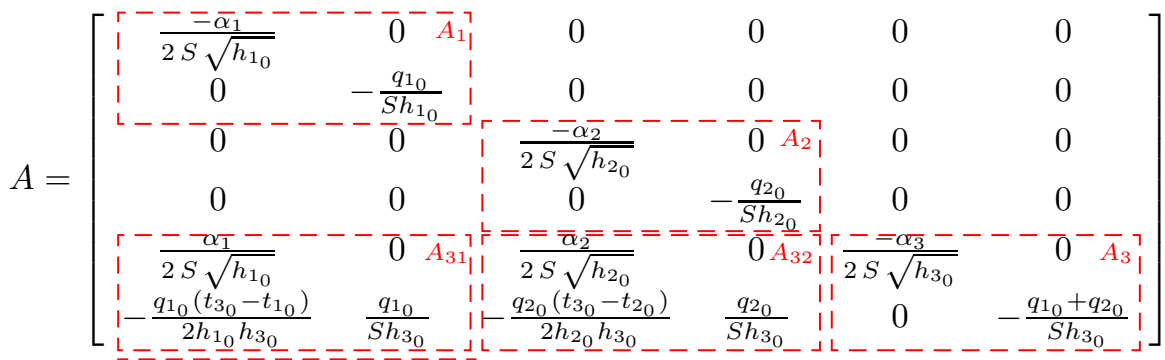

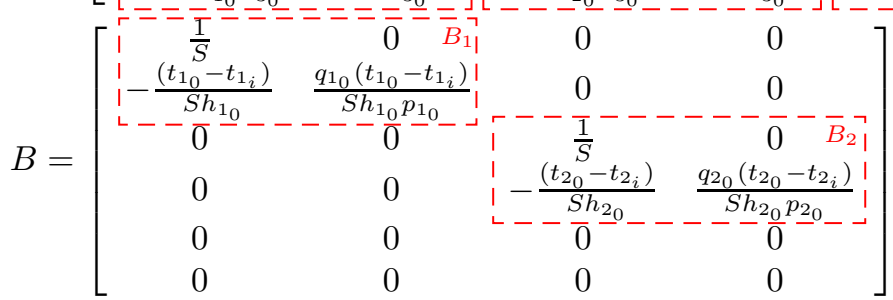

\subsection{Décomposition du système}

Le système est composé de trois sous-systèmes $: s_{1}$ : cuve $1, s_{2}:$ cuve 2 et $s_{3}$ : cuve 3. La figure 8 illustre la décomposition physique du système. Quant à la figure 9 , elle illustre la décomposition fonctionnelle du système.

Les objectifs globaux du système sont $\left(h_{3}, t_{3}\right)$ et les objectifs locaux sont $\left(h_{1}, t_{1}\right)$ et $\left(h_{2}, t_{2}\right)$. À partir des valeurs de références globales $\left(h_{3, \text { ref }}, t_{3, \text { ref }}\right)$, le coordinateur permet de définir les valeurs des références $\left(h_{1, r e f}, t_{1, r e f}\right)$ et $\left(h_{2, r e f}, t_{2, r e f}\right)$ des 
deux sous-systèmes locaux $s_{1}$ et $s_{2}$ dans le but d'assurer les objectifs globaux $\left(h_{3}, t_{3}\right)$. Les matrices $A_{1}, A_{2}, A_{3}, A_{31}, A_{32}, B_{1}$ et $B_{2}$ sont données dans la sous-section précédente. $C_{1}, C_{2}$ et $C_{3}$ sont des matrices unitaires de dimension 2 . Notons que $\left(K_{1}, G_{1}\right)$ et $\left(K_{2}, G_{2}\right)$ sont les gains des lois de commande utilisées par les deux sous-systèmes $s_{1}$ et $s_{2}$, nous les présenterons à la sous-section suivante 6.2.

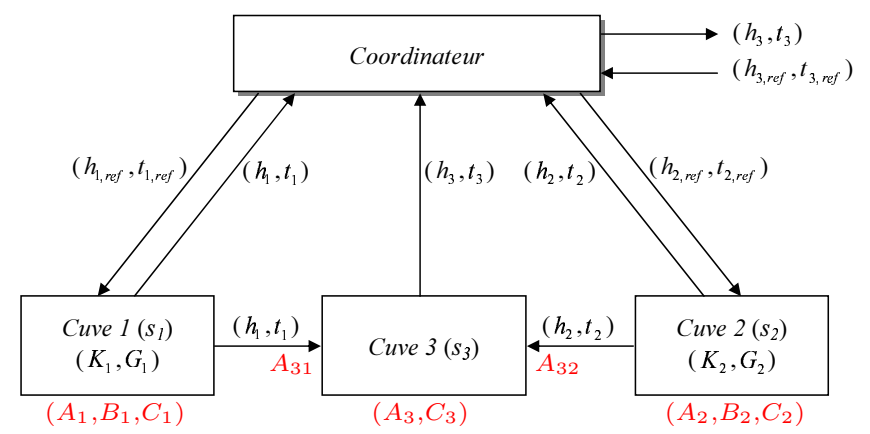

Figure 8. La structure hiérarchique du système

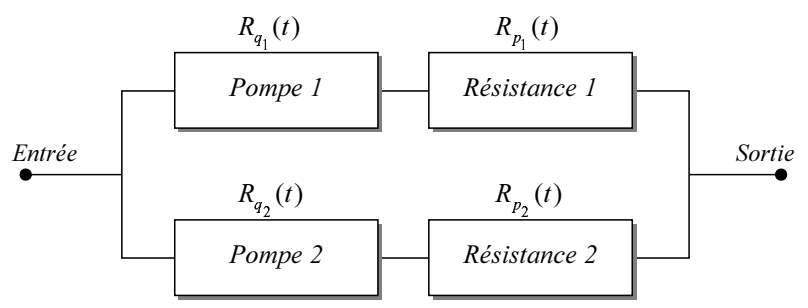

Figure 9. Diagramme de fiabilité du système

Les fiabilités de la pompe 1 , la pompe 2 , la résistance 1 et la résistance 2 sont respectivement notées par $R_{q 1}(t), R_{q 2}(t), R_{p 1}(t)$ et $R_{p 2}(t)$. Notons que la cuve 3 n'est pas représentée car elle ne contient que des composants passifs (cuve, tuyaux...) dont la durée de vie est très grande, donc sa fiabilité est approximée à 1 .

\subsection{Synthèse des régulateurs}

Les valeurs numériques des différents paramètres sont :

$S=0,0154, c=4180, \mu=1000, \alpha_{1}=\alpha_{2}=2,1082 * 10^{-5}, \alpha_{3}=4,2164 * 10^{-5}$, $t_{i_{1}}=15^{\circ} \mathrm{C}, t_{i_{2}}=20^{\circ} \mathrm{C}, t_{1, \min }=15^{\circ} \mathrm{C}, t_{2, \min }=20^{\circ} \mathrm{C}, t_{1, \max }=t_{2, \max }=40^{\circ} \mathrm{C}$, $h_{1, \min }=h_{2, \min }=0 \mathrm{~m}, h_{1, \max }=h_{2, \max }=1 \mathrm{~m}, p_{1, \max }=p_{2, \max }=500 \mathrm{watt}$, $q_{1, \max }=q_{2, \max }=0,0003 \mathrm{~m}^{3} / \mathrm{s}$.

Les sous-systèmes $s_{1}$ et $s_{2}$ sont symétriques, nous présentons uniquement le multimodèle du $s_{1}$, la représentation du $s_{2}$ s'effectue de la même manière. Le domaine de fonctionnement souhaité de la température et du niveau du liquide dans 
la cuve 1 est défini par $t_{1} \in[15,40]$ et $h_{1} \in[0,1]$. Chacun des intervalles est divisé en quatre domaines de validité. Les points de fonctionnement de la température sont $t_{1_{0}}=[18,125,24,375,30,625,36,875]$ et du niveau du liquide sont $h_{1_{0}}=[0,125,0,375,0,625,0,875]$, par conséquent seize modèles locaux, notés $\sum_{1_{(i, j)}}$, avec seize points de fonctionnement sont considérés pour représenter le soussystème. Les points de fonctionnement $q_{1_{0}}(i)$ et $p_{1_{0}}(i, j)$ sont déterminés à partir des valeurs $h_{1_{0}}(i), t_{1_{0}}(j)$ et des équations [57] et [58]. Un modèle $\sum_{1_{(i, j)}}$ est obtenu en remplaçant les valeurs des points de fonctionnement dans les matrices $A_{1}, B_{1}$ et $C_{1}$.

La représentation multimodèle du sous-système $s_{1}$ s'écrit alors :

$$
\left\{\begin{array}{l}
\dot{x}_{1}(t)=\sum_{i, j}^{4} \omega_{i, j}\left(h_{1}(t), t_{1}(t)\right)\left(A_{1_{(i, j)}} x_{1}(t)+B_{1_{(i, j)}} u_{1}(t)\right) \\
y_{1}(t)=\sum_{i, j}^{4} \omega_{i, j}\left(h_{1}(t), t_{1}(t)\right)\left(C_{1_{(i, j)}} x_{1}(t)\right) \\
\omega_{i, j}\left(h_{1}(t), t_{1}(t)\right)=\frac{\mu_{i, j}\left(h_{1}(t), t_{1}(t)\right)}{\sum_{i, j}^{4} \mu_{i, j}\left(h_{1}(t), t_{1}(t)\right)}
\end{array}\right.
$$

avec $x_{1}(t)=\left[h_{1}(t), t_{1}(t)\right]^{T}, \quad u_{1}(t)=\left[q_{1}(t), p_{1}(t)\right]^{T}$ et $y_{1}(t)=\left[h_{1}(t), t_{1}(t)\right]^{T}$

La fonction $\mu_{i, j}\left(h_{1}(t), t_{1}(t)\right)$ est donnée classiquement par le produit des fonctions gaussiennes : $\mu_{i}\left(t_{1}(t)\right)$ qui dépend de $t_{1}(t)$ et $\mu_{j}\left(h_{1}(t)\right)$ qui dépend de $h_{1}(t)$.

Pour chacun des modèles linéaires locaux, nous réalisons la synthèse d'une loi de commande par retour d'état $u_{1_{(i, j)}}(t)=-K_{1_{(i, j)}} x_{1}(t)+G_{1_{(i, j)}} r_{1}(t)$. De la même manière que le multimodèle, l'expression de la loi de commande globale du soussystème $s_{1}$ s'écrit alors : $u_{1}(t)=\sum_{i, j}^{4} \omega_{i, j}\left(h_{1}(t), t_{1}(t)\right) u_{1_{(i, j)}}(t)$.

Les gains $K_{1_{(i, j)}}$ (et $K_{2_{(i, j)}}$ ) permettent d'assurer une dynamique en imposant au sous-système $s_{1}$ (et au $s_{2}$ ) en boucle fermée deux pôles $p_{1}$ et $p_{2}$. Tandis que les gains $G_{1_{(i, j)}}\left(\right.$ et $G_{2_{(i, j)}}$ ) permettent de satisfaire les contraintes en poursuite de références.

Afin d'avoir une erreur statique nulle, nous introduisons une action intégrale entre la mesure $y_{1}$ et la référence $r_{1}$ et nous utilisons la forme augmentée du sous-système. Par conséquent, la synthèse de la loi de commande consiste à déterminer les gains $K_{1_{(i, j)}}$ et $G_{1_{(i, j)}}$ permettant d'imposer les pôles $p_{1}$ et $p_{2}$ et deux pôles supplémentaires $p_{3}$ et $p_{4}$ pour l'intégral choisis de telle sorte qu'ils n'affectent pas la dynamique du sous-système non augmenté. La nouvelle formulation des lois de commande est : $u_{1_{(i, j)}}(t)=-\left[K_{1_{(i, j)}} G_{1_{(i, j)}}\right] x_{1}^{a u g}(t) \quad$ avec $\quad x_{1}^{a u g}(t)=\left[x_{1}(t) \quad \int\left(r_{1}(t)-y_{1}(t)\right)\right]^{T}$

Dans le cas défaillant, un ensemble de matrice $\mathcal{M}_{1}$ (et $\left.\mathcal{M}_{2}\right)$ est défini de façon que $\forall M^{a u g} \in \mathcal{M}_{1}$, les pôles de $M^{a u g}$ ont des valeurs de $\pm 10 \%$ des pôles nominaux. Pour application numérique, nous souhaitons avoir des pôles $p_{1}=-0,0015, p_{2}=$ $-0,0088, p_{3}=-0,0001$ et $p_{4}=-0,0001$. Les nouveaux gains optimaux $K_{1_{(i, j)}^{\text {opt }}}$ et $G_{1_{(i, j)} \text { opt }}$ de chacun des modèles locaux $\sum_{1_{(i, j)}}$ du sous-système $s_{1}$ sont calculés en utilisant la méthode pseudo-inverse avec les modifications de la sous-section 4.3 . 


\subsection{Définition des structures}

Afin d'illustrer la méthode, nous considérons que le défaut simulé consiste en une perte de puissance de $\beta^{f} \%$ de la résistance de chauffe de la cuve 1 . Une structure nominale et trois structures en présence de défaut sont définies hors ligne.

En l'absence de défauts, la structure nominale du système, notée $\mathcal{S}^{\text {nom }}$, exploite tous les sous-systèmes (les trois cuves).

Dans la structure $\mathcal{S}^{1}$, le système utilise uniquement la cuve 2 et 3 . La cuve 1 n'est pas exploitée. Dans ce cas, les objectifs globaux $\left[h_{3}(t), t_{3}(t)\right]$ dépendent uniquement des objectifs locaux $\left[h_{2}(t), t_{2}(t)\right]$ sans tenir compte de $\left[h_{1}(t), t_{1}(t)\right]$. Soit :

$$
\left\{\begin{aligned}
h_{3} & =\left(\frac{\alpha_{2} \sqrt{h_{2}}}{\alpha_{3}}\right)^{2} \\
t_{3} & =\frac{\alpha_{2} t_{2} \sqrt{h_{2}}}{\alpha_{3} \sqrt{h_{3}}}
\end{aligned}\right.
$$

Dans la structure $\mathcal{S}^{2}$, la résistance 1 est exploitée au maximum de sa capacité restante $p_{1}=\left(1-\beta^{f}\right) \cdot p_{1, \max }$. Les équations du système au régime statique deviennent :

$$
\left\{\begin{aligned}
h_{3} & =\left(\frac{\alpha_{1} \sqrt{h_{1}}+\alpha_{2} \sqrt{h_{2}}}{\alpha_{3}}\right)^{2} \\
t_{3} & =\frac{\alpha_{1} t_{1}\left(\left(1-\beta^{f}\right) \cdot p_{1, \max }\right) \sqrt{h_{1}}+\alpha_{2} t_{2} \sqrt{h_{2}}}{\alpha_{3} \sqrt{h_{3}}}
\end{aligned}\right.
$$

Dans la dernière structure $\mathcal{S}^{3}$, le système utilise la totalité de ses sous-systèmes avec toutes les combinaisons possibles des objectifs locaux en termes de références locales $\left[h_{1, r e f}(t), t_{1, \text { ref }}(t)\right]$ et $\left[h_{2, r e f}(t), t_{2, r e f}(t)\right]$. Les références sont données par :

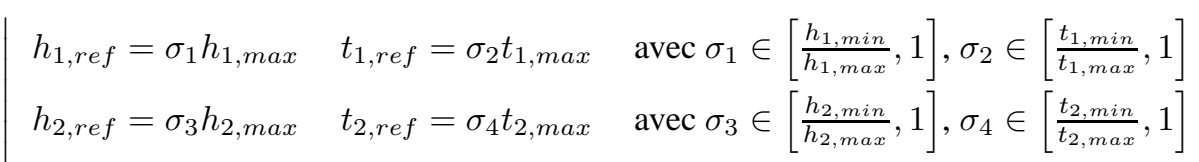

\subsection{Résultats et commentaires}

Dans cette sous-section, nous illustrons les résultats théoriques à l'aide de simulations. Dans les scénarios simulés, les objectifs globaux souhaités (références) sont : $h_{3, \text { ref }}=0,2 \mathrm{~m}$ et $t_{3, \text { ref }}=21^{\circ} \mathrm{C}$. La limite de fiabilité désirée à l'instant $t_{e}$ est $R^{*}=0,65$. L'instant d'apparition du défaut est $t_{f}=3500 \mathrm{~s}$ et l'instant de la renconfiguration est $t_{r}=3510 \mathrm{~s}$, en supposant un retard à la détection de $10 \mathrm{~s}$.

\section{Cas du système nominal}

Pour illustrer les performances de l'approche multimodèle proposée, nous supposons que les objectifs locaux $\left(h_{1, r e f}, t_{1, r e f}\right)$ et $\left(h_{2, r e f}, t_{2, r e f}\right)$ prennent plusieurs valeurs $\left(0,2 \mathrm{~m}, 21{ }^{\circ} \mathrm{C}\right),\left(0,2 \mathrm{~m}, 21{ }^{\circ} \mathrm{C}\right)$ entre $t_{0}=0 \mathrm{~s}$ et $t=2000 \mathrm{~s}$ et 
$\left(0,25 \mathrm{~m}, 22,5{ }^{\circ} \mathrm{C}\right),\left(0,1555 \mathrm{~m}, 19,1{ }^{\circ} \mathrm{C}\right)$ durant l'intervalle $[2000 \mathrm{~s}, 5000 \mathrm{~s}]$ et $\left(0,15 \mathrm{~m} 20^{\circ} \mathrm{C}\right),\left(0,257 \mathrm{~m}, 21,75^{\circ} \mathrm{C}\right)$ de $t=5000 \mathrm{~s}$ jusqu'à $t=7000 \mathrm{~s}$.

La figure 10 fait apparaître l'ensemble des signaux de commandes $q_{1}, p_{1}, q_{2}$ et $p_{2}$ et de mesures $h_{1}, h_{2}, t_{1}$ et $t_{2}$ des sous-systèmes locaux (cuve 1 et cuve 2 ) tandis que la figure 11 représente les objectifs globaux du système $h_{3}$ et $t_{3}$. Les changements des valeurs de références $h_{1, \text { ref }}, t_{1, \text { ref }}, h_{2, \text { ref }}$ et $t_{2, \text { ref }}$ à $t=2000 \mathrm{~s}$ et $t=5000 \mathrm{~s}$ conduisent à des changements aux niveaux des commandes $q_{1}, p_{1}, q_{2}$ et $p_{2}$.

Nous remarquons également qu'il y a des dérives au niveau de $h_{3}$ et $t_{3}$ aux instants $t=2000 \mathrm{~s}$ et $t=5000 \mathrm{~s}$ dues aux changements des valeurs de références.
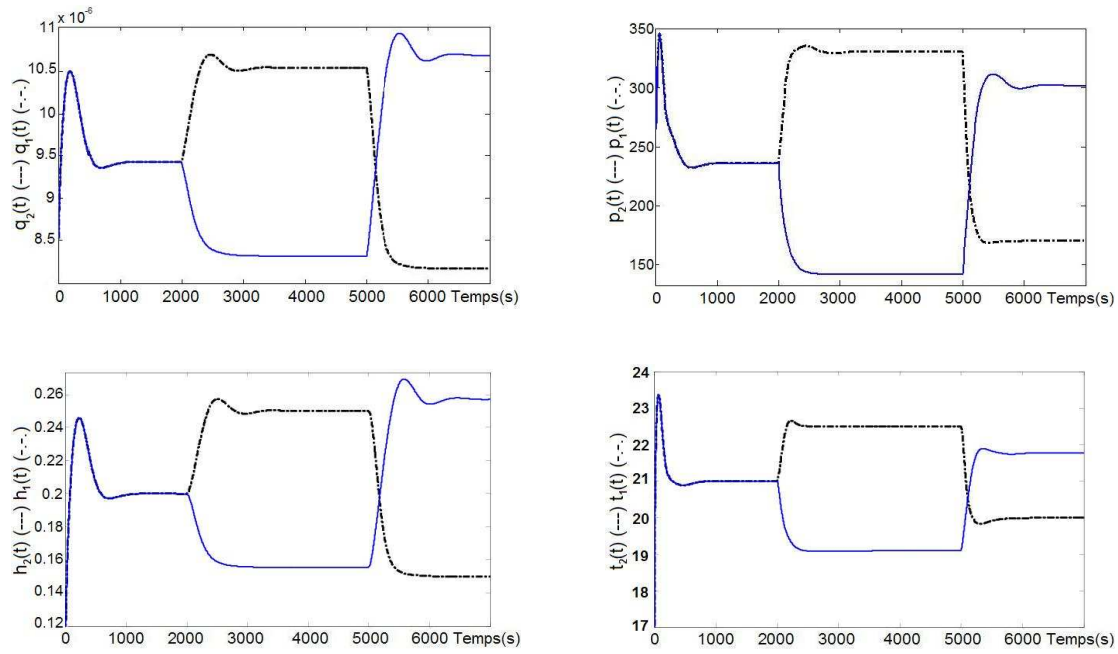

Figure 10. Commandes et sorties de la cuve 1 et 2 dans le cas nominal
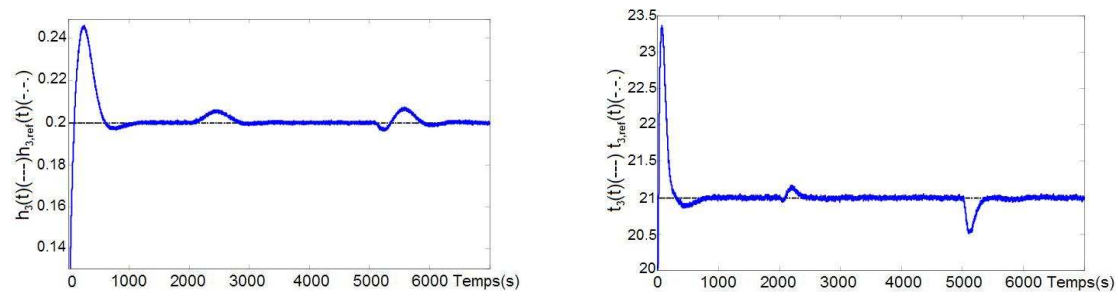

Figure 11. Les objectifs globaux $h_{3}$ et $t_{3}$

Les changements des références permettent d'illustrer l'efficacité des lois de commande synthétisées pour les modèles locaux définis dans la section multimodèle. Notons que durant cette simulation, plusieurs modèles locaux sont activés suivant les valeurs des sorties, les objectifs globaux, $h_{3}$ et $t_{3}$ rejoignant les valeurs de références $h_{3, \text { ref }}=0,2 \mathrm{~m}$ et $t_{3, \text { ref }}=21^{\circ} \mathrm{C}$ comme illustré à la figure 11. 
Cas du système défaillant $\beta^{f}=40 \%$

Notons que le défaut actionneur considéré lors des simulations est de type biais et a une amplitude de $\beta^{f}=40 \%$ de la commande $p_{1}$. Il apparait à l'instant $t_{f}=3500 \mathrm{~s}$. La période de fonctionnement désirée après l'apparition du défaut est $T_{d}=10000 \mathrm{~h}$.

Nous montrons sur la figure 12 l'effet du défaut actionneur de la puissance $p_{1}$ sur le niveau et la température du liquide dans la cuve 1 de part la régulation. Le défaut agit directement sur la température $t_{1}$, mais il n'influence pas le niveau $h_{1}$. La figure 13 illustre l'effet du défaut sur les objectifs globaux, puisqu'ils dépendent directement de l'ensemble des objectifs locaux. Nous remarquons qu'après un certain temps la température du liquide rejoint la référence, cela est dû à l'action intégrale mise entre la mesure et la référence, mais le défaut existe (figure 12).
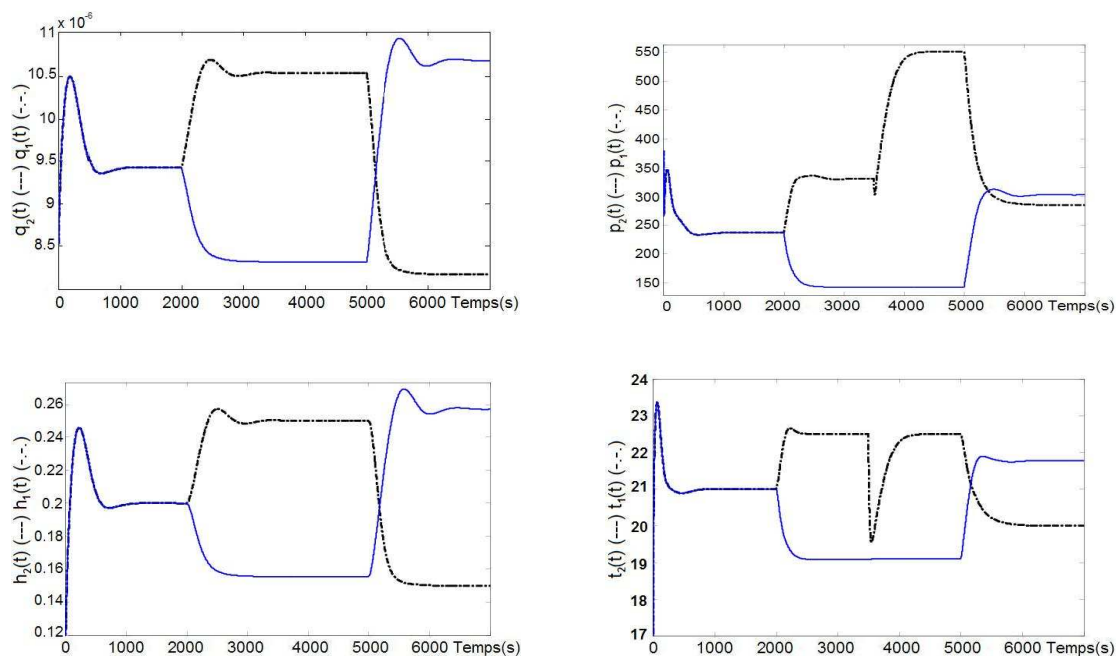

Figure 12. Commandes et sorties de la cuve 1 et 2 dans le cas défaillant $\beta^{f}=40 \%$
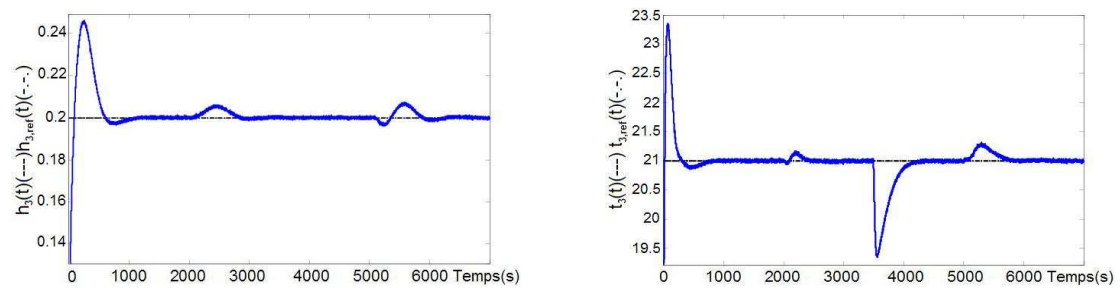

Figure 13. Les objectifs globaux $h_{3}$ et $t_{3}$ dans le cas défaillant $\beta^{f}=40 \%$

L'effet du défaut peut être remarqué également au niveau des valeurs propres du système, les valeurs propres de chacun des modèles locaux de la cuve 1 sont données par $[-0,00577-0,001119-0,0001524-0,000134]$. Rappelons que les valeurs propres du sous-système nominal sont $[-0,0088-0,0015-0,0001-0,0001]$. Nous pouvons constater qu'il y a des écarts, ce qui influe la dynamique du système. 


\section{Cas du système reconfiguré $\beta^{f}=40 \%$}

Le tableau 1 présente les valeurs des objectifs locaux $h_{1}, t_{1}, h_{2}$ et $t_{2}$ ainsi que les objectifs globaux $h_{3}$ et $t_{3}$ du système utilisant les trois structures $\mathcal{S}^{1}, \mathcal{S}^{2}$ et $\mathcal{S}^{3}$. Les fiabilités des sous-systèmes et du système complet, les indices de performances statiques et dynamiques sont également présentés dans le tableau.

L'indice $\mathcal{J}^{m}$ est la somme de $J_{\text {statique }}$ et $J_{d y n}$ chacun pondéré par un poids $\rho$ définis par l'équation [55]. Dans cet exemple les poids sont égaux et $\rho=0.5$.

Nous remarquons que les structures $\mathcal{S}^{2}$ et $\mathcal{S}^{3}$ respectent les contraintes de fiabilité $R^{*}=0.65$. La structure $\mathcal{S}^{3}$ est sélectionnée optimale car son indice de performances $\mathcal{J}^{3}$ est inférieur que $\mathcal{J}^{2}$.

\begin{tabular}{|c|c|c|c|}
\hline & $\mathcal{S}^{1}$ & $\mathcal{S}^{2}$ & $\mathcal{S}^{3}$ \\
\hline$h_{1}$ & - & 0.0990 & 0.1932 \\
$t_{1}$ & - & 25.818 & 19.4647 \\
$h_{2}$ & 0.8 & 0.3361 & 0.2068 \\
$t_{2}$ & 20.978 & 18.241 & 22.4729 \\
\hline$R_{q_{1}}$ & - & 0.92 & 0.9074 \\
$R_{p_{1}}$ & - & 0.49 & 0.7836 \\
$R_{q_{2}}$ & 0.76 & 0.89 & 0.9155 \\
$R_{p_{2}}$ & 0.79 & 0.95 & 0.8367 \\
\hline$h_{3}$ & 0.2 & 0.2 & 0.2 \\
$t_{3}$ & 20.978 & 20.907 & 21 \\
$J_{\text {statique }}$ & 0.001 & 0.0044 & $5.6296 * 10^{-4}$ \\
$J_{d y n}$ & 0 & $8.5321 * 10^{-5}$ & $8.5321 * 10^{-5}$ \\
$\mathcal{J}_{m}^{m}$ & $5.0 * 10^{-4}$ & $2.2426 * 10^{-3}$ & $3.2414 * 10^{-4}$ \\
$R_{g}\left(T_{d}\right)$ & 0.60 & 0.9185 & 0.9324 \\
\hline
\end{tabular}

Tableau 1. Performances globales et locales du système selon $\mathcal{S}^{1}, \mathcal{S}^{2}$ et $\mathcal{S}^{3}\left(\beta^{f}=40 \%\right)$

La figure 15 fait apparaître les commandes $q_{1}, q_{2}$ et $p_{1}, p_{2}$ corrependant aux valeurs d'objectifs locaux $h_{1}, h_{2}$ et $t_{1}, t_{2}$ qui changent des valeurs nominales aux valeurs indiquées par le tableau 1. Les sorties $h_{1}, h_{2}$ et $t_{1}, t_{2}$ suivent respectivement les références $0,1932 \mathrm{~m}, 0,2068 \mathrm{~m}$ et $19,4647{ }^{\circ} \mathrm{C}, 22,4729{ }^{\circ} \mathrm{C}$ conformément aux valeurs de la structure $\mathcal{S}^{3}$ indiquées au tableau 1 . Ces nouvelles valeurs d'objectifs locaux permettent d'atteindre les objectifs globaux comme l'illustre la figure 14. Une dérive au niveau de $h_{3}$ peut être remarquée lors de la validation de la nouvelle structure due au changement des valeurs de références $h_{1}$ et $h_{2}$ dépendant de la dynamique des deux cuves 1 et 2 . Pour la même raison, une dérive existe au niveau de la température $t_{3}$ mais de manière moins significative qu'en l'absence reconfiguration.
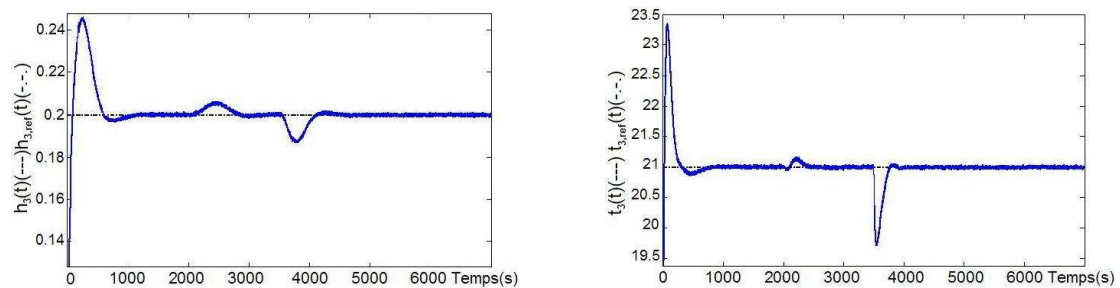

Figure 14. Les objectifs globaux $h_{3}$ et $t_{3}$ du système reconfiguré $\left(\beta^{f}=40 \%\right)$ 

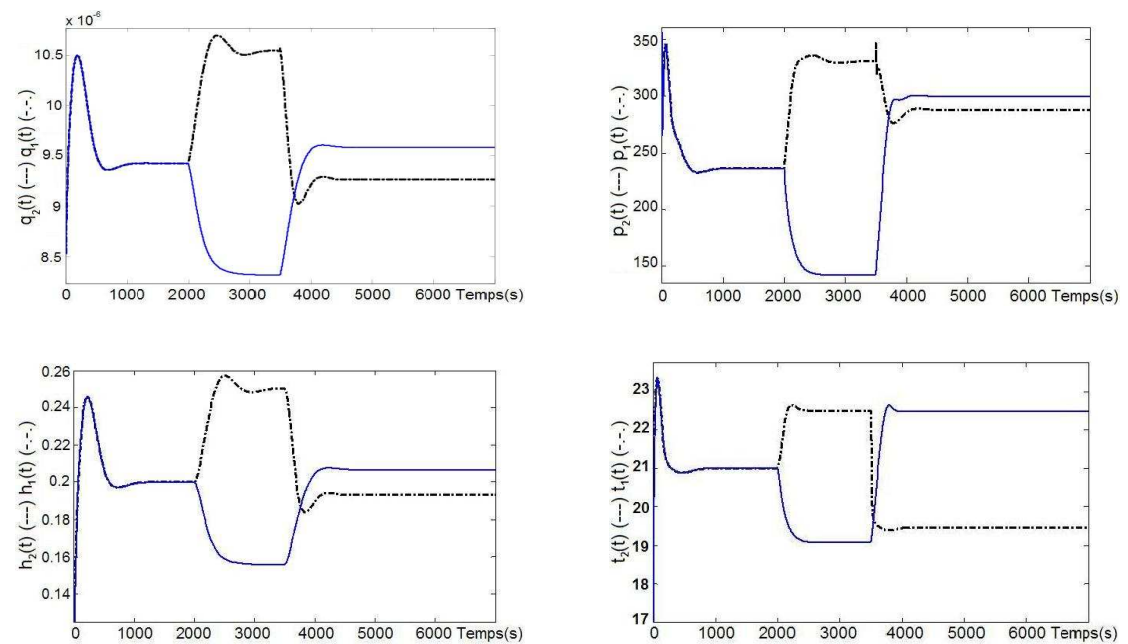

Figure 15. Commandes et sorties de la cuve 1 et 2 du système reconfiguré $\left(\beta^{f}=40 \%\right)$

\section{Conclusion}

Cet article présente une stratégie de la commande tolérante aux défauts conduisant à la détermination d'une nouvelle structure du système, lorsqu'un défaut apparaît. En présence d'un défaut, les objectifs du système ne sont pas atteignables en utilisant la structure nominale, la méthode proposée permet de conduire le système vers une autre structure en redéfinissant des nouvelles performances locales de chaque sous-système afin de garder les performances globales, ou au moins des performances dégradées acceptables. Notre approche est basée sur l'analyse de la fiabilité de certains composants du système. Le diagramme de fiabilité a été utilisé pour obtenir la décomposition fonctionnelle permettant de fournir les formules utilisées pour calculer la fiabilité du système complet à partir des fiabilités des sous-systèmes. Notons que les composants ayant des taux de défaillance non significatifs ne sont pas introduits dans l'analyse de la fiabilité. Cependant, il est possible de les introduire dans ce travail et ils seront traités de la même manière que les composants ayant des taux de défaillance élevés.

\section{Bibliographie}

AFNOR N., « Terminologie relative à la fiabilité - maintenabilité - disponibilité », $N F X, 60$ 500,1988

Bonivento C., Capiluppi M., Marconi L., Paoli A., Rossi C., « Reliability evaluation for fault diagnosis in complex systems », 6th IFAC Safeprocess, Beijing, PR China., 2006.

Bonivento C., Marconi L., Paoli A., Rossi C., «A framework for reliability analysis of complex diagnostic systems », 5th IFAC Safeprocess, Washington DC, USA, 2003.

Chen J., Patton R., Robust model-based fault diagnosis for dynamic systems, Kluwer Academic Publishers, Norwell, MS, 1999.

Cox D., « Regression models and life tables », Journal of the Royal Statistical Society, vol. 34, p. 187-220, 1972.

Cox D., Oakes D., Analysis of survival data, Chapman and Hall, 1984. 
Figueras J., Puig V., Quevedo J., « Multiple fault diagnosis system design using reliability analysis : Application to Barcelona rain-gauge network », 6th IFAC Safeprocess, Beijing, 2006.

Finkelstein M. S., «A note on some aging properties of the accelerated life model », Reliability Engineering and System Safety, vol. 71, issue 1, p. 109-112, 1999.

Frank P., «Fault diagnosis in dynamic systems using analytical and knowledge-based reduncancy - a survey », Automatica, vol. 26, n 3, p. 459-474, 1990.

Gao Z., Antsaklis P., « Pseudo-Inverse Methods for reconfigurable control with guaranteed stability », 11th IFAC World Congress, p. 293-298, 1990.

Gao Z., Antsaklis P., « Stability of the Pseudo-Inverse Method for reconfigurable control systems », International Journal of Control, vol. 53, n 3, p. 717-729, 1991.

Guenab F., «Contribution aux systèmes tolérants aux défauts : Synthèse d'une méthode de reconfiguration et/ou de restructuration intégrant la fiabilité des composants », Thèse de doctorat, Nancy-Université, France, 2007.

Guenab F., Theilliol D., Weber P., Ponsart J., Sauter D., « Fault Tolerant Control Method Based on Cost and Reliability Analysis », 16th IFAC World Congress, Czech Republic, 2005.

Guenab F., Theilliol D., Weber P., Zhang Y., Sauter D., « Fault Tolerant Control System Design : A Reconfiguration Strategy Based on Reliability Analysis Under Dynamic Behavior Constraints », 6th IFAC Symposium Safeprocess, Beijing, PR China, 2006.

Huang C. Y., Stengel R., « Restructuable control using proportional-integral implicit model following », Journal of Guidance, Control and Dynamics, vol. 13, n 2, p. 303-309, 1990.

Isermann R., « Process fault detection based on modelling and estimation methods - a survey », Automatica, vol. 20, n 4, p. 387-404, 1984.

Isermann R., « Supervision, fault detection and fault diagnosis methods - an introduction », Control Eng. Practice, vol. 5, n 5, p. 639-652, 1997.

Li H., Zhao Q., « Reliability evaluation of fault tolerant control systems with semi-markov FDI model », 6th IFAC Symposium Safeprocess, Beijing, PR China, 2006.

Martorell S., Sanchez A., Serradell V., « Age-dependent reliability model considering effects of maintenance and working conditions », Reliability Engineering and System Safety, 1999.

Murray-Smith R., Johansen T., Multiple model approaches to modelling and control, Taylor and Francis, 1997.

Ostroff A., « Techniques for accommodating control effector failures on a mildly statically unstable airplane », American Control Conference, Boston, IEEE, p. 906-913, 1985.

Patton R., Chen J., Nielsen S. B., « Model-based methods for fault diagnosis : Some guidlines », Trans. of the institute of Measurement and Control, vol. 17, p. 73-81, 1995.

Staroswiecki M., «A structural framework for the design of FDI system in large scale industrial plants », 'Issues of Fault tolerant diagnosis for dynamic system'. Springer-Verlag, 2000.

Staroswiecki M., "Fault tolerant control : the pseudo-inverse method revisited », Proc. 16th IFAC World Congress, Prague, Czech Republic, 2005.

Staroswiecki M., Hoblos G., Aitouche A., « Sensor network design for fault tolerant estimation », International journal of adaptive control and signal processing, 2004.

Walker B., «Fault tolerant control system reliability and performance prediction using semimarkov models », Proc. IFAC Safeprocess'97, Hull, England, p. 1056-1067, 1997.

Wu N., « Reliability of fault tolerant control systems : Part I », IEEE Conference on Decision and Control, 2001a.

Wu N., «Reliability of fault tolerant control systems : Part II », IEEE Conference on Decision and Control, 2001b.

Wu N., «Coverage in fault tolerant control », Automatica, vol. 40, issus 4, p. 537-548, 2004.

Wu N., Wang X., Smapath M., Kott G., « An operational approach to buget-constrainted reliability allocation », 15th IFAC World Congress, Barcelona, Spain, p. 199-204, 2002. 